\title{
Distinct fastigial output channels and their impact on temporal lobe seizures
}

\author{
Martha L. Streng ${ }^{1}$, Madison Tetzlaff ${ }^{1}$, Esther Krook-Magnuson ${ }^{1}$ \\ ${ }^{1}$ Department of Neuroscience, University of Minnesota, Minneapolis, MN, USA. \\ Correspondence: $\quad$ Martha Streng, Ph.D. \\ Department of Neuroscience \\ University of Minnesota \\ Jackson Hall, room 6-145 \\ 321 Church Street SE \\ Minneapolis, MN 55455 \\ Phone: 612-624-4494 \\ Email: stren021@umn.edu
}

Running title: Distinct fastigial outputs control seizures

Manuscript properties:

Number of pages: 56

Number of figures: 10

Word counts
Abstract: 250
Significance statement: 120
Introduction: 971
Discussion: 2413

The authors declare no competing financial interests. 


\section{Acknowledgments}

Dr. Michael Benneyworth and the University of Minnesota Mouse Behavioral Core and Dr. Erin Larson and the University of Minnesota Optogenetics Core provided technical assistance and equipment. Laurel Schuck, Susan Khanpour, Madeline Marker, Jacob Weiner, Thai Loyd, Jack Redepenning, Isaac Hoff, Jane Yap, and Kayla Togneri of the Krook-Magnuson lab provided technical assistance. 


\section{Abstract}

2 Despite being canonically considered a motor control structure, the cerebellum is increasingly

3 recognized for important roles in processes beyond this traditional framework, including seizure

4 suppression. Excitatory fastigial neurons project to a large number of downstream targets, and it

5 is unclear if this broad targeting underlies seizure suppression, or if a specific output may be

6 sufficient. To address this question, we used the intrahippocampal kainic acid mouse model of

7 temporal lobe epilepsy, male and female animals, and a dual-virus approach to selectively label

8 and manipulate fastigial outputs. We examined fastigial neurons projecting to the superior

9 colliculus, medullary reticular formation, and central lateral nucleus of the thalamus, and found

10 that these comprise largely non-overlapping populations of neurons which send collaterals to

11 unique sets of additional thalamic and brainstem regions, creating distinct, somewhat

12 overlapping, "output channels". We found that neither optogenetic stimulation of superior

13 colliculus nor reticular formation output channels attenuated hippocampal seizures. In contrast,

14 on-demand stimulation of fastigial neurons targeting the central lateral nucleus robustly inhibited

15 seizures. Our results indicate that fastigial control of hippocampal seizures does not require

16 simultaneous modulation of many fastigial output channels. Rather, selective modulation of the

17 fastigial output channel to the central lateral thalamus, specifically, is sufficient for seizure

18 control. This may provide a means for more selective therapeutic interventions, which provide

19 seizure control while minimizing unwanted side effects. More broadly, our data highlight the

20 concept of specific cerebellar output channels, whereby discrete cerebellar nucleus neurons

21 project to specific aggregates of downstream targets, with distinct functional outcomes. 


\section{Significance statement}

25 The cerebellum has an emerging relationship with non-motor systems and may represent a

26 powerful target for therapeutic intervention in temporal lobe epilepsy. We find that fastigial

27 neurons project to numerous brain regions via largely segregated output channels, and that

28 excitation of fastigial neurons projecting to the central lateral nucleus of the thalamus, but not the

29 superior colliculus or reticular formation, is sufficient to attenuate hippocampal seizures. These

30 findings illustrate an important conceptual framework: fastigial neurons project to aggregates of

31 downstream targets via distinct output channels, which cannot be predicted simply by somatic

32 location within the nucleus, and these channels have distinct functional outcomes. This nuanced

33 appreciation of fastigial outputs may provide a path for therapeutic interventions with minimized

34 side effects. 


\section{INTRODUCTION}

37 The cerebellum contains more than half of all total neurons in the central nervous system

38 (Andersen et al., 1992), accounts for as much as 20\% of the total oxygen consumption of the

39 brain (Howarth et al., 2010), and is reciprocally connected with a large number of cortical and

40 subcortical regions (Ramnani, 2006; Strick et al., 2009; Salmi et al., 2010; Ramnani, 2012;

41 Kipping et al., 2013). Though canonically considered a motor control structure, mounting

42 evidence indicates that the cerebellum is heavily involved in functions beyond this traditional

43 framework (Schmahmann, 1996; Hilber et al., 1998; Leggio et al., 1999; Colombel et al., 2004;

44 Popa et al., 2014; Yu and Krook-Magnuson, 2015; Schmahmann, 2019; Shipman and Green,

45 2019). Recent work has shown that the cerebellum can profoundly influence hippocampal

46 function, with the ability to modulate hippocampal neuronal dynamics (Choe et al., 2018; Zeidler

47 et al., 2020) and alter hippocampal-dependent behavior (Rochefort et al., 2011; Lefort et al.,

48 2019; Zeidler et al., 2020). The influence of cerebellar dynamics on hippocampal function, in

49 addition to being a topic of great scientific interest, has the potential to meet an urgent

50 translational need in temporal lobe epilepsy (TLE), a disorder characterized by chronic,

51 spontaneous seizures typically arising in the hippocampal formation. TLE is the most common

52 form of epilepsy in adults, but current treatment options have limited efficacy and carry the

53 potential for problematic side effects, leaving 30-40\% of epilepsy patients with uncontrolled

54 seizures (England et al., 2012). We recently demonstrated that even very brief optogenetic

55 interventions delivered to excitatory neurons in the cerebellar fastigial nucleus was highly

56 effective at terminating hippocampal seizures in a mouse model of TLE (Streng and Krook-

57 Magnuson, 2020b).

58

59 While recent work indicates a lack of a direct, mono-synaptic, connections between the

60 cerebellum and the hippocampus, at least in rodents (Rochefort et al., 2013; Bohne et al., 2019;

61 Krook-Magnuson, 2020), the fastigial nucleus does project to over 60 downstream targets (Fujita 
62 et al., 2020). This large number of output targets raises the possibility that seizure suppression

63 via fastigial excitation requires the coordinated modulation of many areas (Eelkman Rooda et al.,

64 2021). Alternatively, a specific output may be sufficient to inhibit seizures, and previous work

65 suggests at least some degree of segregation of cerebellar outputs (Noda et al., 1990; Fuchs et al.,

66 1993; Teune et al., 2000; Zhang et al., 2016; Fujita et al., 2020). Fastigial targets of potential

67 interest for hippocampal seizure control include the thalamus, superior colliculus, and the

68 reticular formation (Angaut and Bowsher, 1970; Batton et al., 1977; Bentivoglio and Kuypers,

69 1982; Andrezik et al., 1984; Angaut et al., 1985).

70

71 The cerebellum has numerous connections with thalamocortical networks (Middleton and Strick,

72 1998), and the fastigial nucleus in particular projects to several distinct thalamic nuclei (Haroian

73 et al., 1981; Fujita et al., 2020). Deep brain stimulation (DBS) trials targeting the thalamus

74 (albeit the anterior nucleus) have reduced seizures for some patients (Salanova et al., 2015) and

75 activation of neurons in the deep cerebellar nuclei disrupt spike-and-wave discharges observed

76 during thalamocortical absence seizures (Kros et al., 2015; Eelkman Rooda et al., 2021).

77 Cerebellar connections to intralaminar and midline thalamic nuclei may be especially relevant for

78 TLE, as they have been implicated in regulating limbic seizures (Wicker and Forcelli, 2016; Feng

79 et al., 2017). While much of the current seizure literature has focused on the central lateral (CL)

80 thalamus in the context of consciousness (Gummadavelli et al., 2015; Kundishora et al., 2017; Xu

81 et al., 2020), the CL is of particular interest to us in the context of cerebellar mediated seizure

82 suppression: its activity is depressed (and bursty) during focal limbic seizures (Feng et al., 2017),

83 and it provides a potential route (via the anterior cingulate) from the cerebellum to the

84 hippocampus (Van der Werf et al., 2002; Rajasethupathy et al., 2015).

85

86 Another candidate region of interest is the superior colliculus (SC), which in addition to receiving

87 fastigial input (Roldan and Reinoso-Suarez, 1981; Fujita et al., 2020) is proposed to play a role in 
88 regulating seizure activity (Garant and Gale, 1987; Dean and Gale, 1989; Weng and Rosenberg,

89 1992), and can inhibit seizures in multiple animal models (Gale et al., 1993; Soper et al., 2016).

90 The fastigial nucleus also has extensive projections to the reticular formation (Andrezik et al.,

91 1984; Zhang et al., 2016; Fujita et al., 2020), a collection of nuclei important for controlling brain

92 states (Moruzzi and Magoun, 1949; Jones, 2003) which could potentially underlie seizure control

93 (Ewell et al., 2015; Khan et al., 2018; Purnell et al., 2018; Streng and Krook-Magnuson, 2020a).

94 Clearly, there are several strong candidate regions for mediating the seizure inhibition seen with

95 on-demand fastigial stimulation, if concurrent excitation of multiple downstream targets is not

96 required.

98 We therefore set out to examine fastigial outputs to the central lateral thalamus, the superior

99 colliculus, and the medullary reticular formation, to determine 1) if these areas are targeted by

100 separate fastigial neurons, 2) if fastigial neurons targeting these areas also target other areas (and

101 if so, which), and 3) if any of these output channels, in isolation, is able to inhibit seizures. Using

102 a dual viral targeting strategy, we labeled populations of fastigial neurons that project to the CL,

$103 \mathrm{SC}$, or reticular formation. We find that these neurons represent largely distinct populations and

104 project to generally segregating additional downstream areas. Using the intrahippocampal kainate

105 mouse model of temporal lobe epilepsy, and on-demand optogenetic manipulation of specific

106 output pathways upon online detection of spontaneous seizures (Armstrong et al., 2013), we

107 further find that cerebellar control over hippocampal seizures can be achieved via activation of a

108 specific output channel, rather than requiring broader network effects. Specifically, excitation of

109 CL-projecting fastigial neurons, but not the other two output pathways examined, is able to

110 robustly attenuate hippocampal seizures. Together, our results illustrate nuanced fastigial nucleus

111 output channels with important, potentially clinically relevant, different functional consequences. 


\section{MATERIALS AND METHODS}

\section{Ethical approval}

116 All experimental protocols were approved by the University of Minnesota's Institutional Animal

117 Care and Use Committee.

\section{Animals}

120 For all experiments, mice were bred in-house and had ad libitum access to food and water in all

121 housing conditions. Black-6 mice (C57BL/6J; Jackson Laboratory stock 000664) were used for

122 initial examination of broad fastigial projections, for output channel specific labeling and

123 modulation, and for a subset of terminal stimulation experiments. Mice expressing Cre

124 selectively in VGluT2-expressing neurons (Vong et al., 2011) (B6J.129S6(FVB)-

125 Slc17a $6^{\text {tm2(cre)Lowl } / M w a r J ; ~ J a c k s o n ~ L a b o r a t o r y ~ s t o c k ~} 028863$ ) were also utilized for on-demand

126 terminal stimulation experiments.

128 Animals were sexed at the time of weaning on the basis of external genitalia. Both male and

129 female mice were used for all experiments. While experiments were not powered to test for sex

130 differences, no trends of sex differences were observed. Until optical fiber and electrode

131 implantation, animals were housed in standard group housing conditions in the Research Animal

132 Resources animal facility at the University of Minnesota. Following implantation, animals were

133 singly housed, and experiments were performed while housed in investigator managed housing.

134 In all conditions, animals were allowed ad libitum access to food and water, and were on a 12

135 hour light; 12 hour dark (/low red light) cycle.

\section{$137 \quad$ Stereotactic surgeries}

138 Viral targeting 
139 For all experiments, AAV serotype 9 was used for opsin expression in fastigial neurons due to its

140 optimal expression in the fastigial nucleus with no apparent retrograde expression (Streng and

141 Krook-Magnuson, 2020b). All injections were performed in adult mice (postnatal day 45 or

142 later).

144 For initial characterization of fastigial fibers, Black-6 mice were injected with $120 \mathrm{~nL}$ of virus

145 encoding GFP in a Cre-independent manner (AAV9-CAG-GFP, titer of $2 \times 10 \mathrm{e}^{12}$, UNC vector

146 core lot \#AV5221, provided to UNC by Edward Boyden) via a Hamilton Neuros syringe into the

147 left cerebellar fastigial nucleus (6.48 posterior, 0.75 left, $3.7 \mathrm{~mm}$ ventral from bregma) under

148 isoflurane anesthesia.

150 For experiments examining specific fastigial output channels, Black-6 mice were first injected

151 with $120 \mathrm{~nL}$ of a retrograde virus encoding Cre (AAVrg-Efla-mCherry-IRES-Cre, titer of $1.37 \mathrm{x}$

$15210^{\wedge} 13$, Addgene viral prep \# 55632-AAVrg, provided to Addgene by Karl Deisseroth (Fenno et

153 al., 2014)) in either the central lateral nucleus (CL) (1.34 mm posterior, $0.75 \mathrm{~mm}$ right, $3.0 \mathrm{~mm}$

154 ventral from bregma), superior colliculus (SC) (4.0 mm posterior, $1.0 \mathrm{~mm}$ right, $2.5 \mathrm{~mm}$ ventral

155 from bregma), ventral medullary reticular nucleus $(\mathrm{MdV})(7.5 \mathrm{~mm}$ posterior, $0.36 \mathrm{~mm}$ right, 4.0

$156 \mathrm{~mm}$ ventral from bregma), or ventral lateral nucleus (VL) (1.25 mm posterior, $0.96 \mathrm{~mm}$ right, 3.5

$157 \mathrm{~mm}$ ventral from bregma). Following retrograde viral injection, the contralateral fastigial nucleus

158 (6.48 posterior, 0.75 left, $3.7 \mathrm{~mm}$ ventral from bregma) was injected with virus encoding

159 Channelrhodopsin in a cre-dependent manner (AAV9-EF1a-double floxed-hChR2(H134R)-

160 EYFP-WPRE-HGHpA, titer of $2.2 \times 10^{13}$ Addgene viral prep \#202198-AAV9, lot \#V22125,

161 provided to Addgene by Karl Deisseroth)(Gradinaru et al., 2007) consistent with our previously

162 published methods for successful viral targeting of this nucleus (Streng and Krook-Magnuson,

163 2020b). After every injection, the syringe was held in place for a minimum of 10 minutes before

164 being withdrawn. On-demand interventions and/or characterization of fibers were conducted a 
165 minimum of 6 weeks post viral injection. Mice with off target expression were excluded from

166 analyses ( $\mathrm{n}=1$ animal).

168 For experiments targeting fastigial terminals, Black-6 mice were injected with virus encoding

169 Channelrhodopsin fused to green fluorescent protein (AAV9-CAG-ChR2-GFP, titer of 2.1x10e ${ }^{12}$,

170 UNC vector core lot \#AV5406D, provided to UNC by Edward Boyden) or VGluT2-Cre mice

171 were injected with virus encoding Channelrhodopsin in a Cre-dependent manner (AAV9-EF1a-

172 double floxed-hChR2(H134R)-EYFP-WPRE-HGHpA, titer of 2.2x10 ${ }^{13}$, Addgene viral prep

173 \#202198-AAV9, lot \#V22125, provided to Addgene by Karl Deisseroth)(Gradinaru et al., 2007).

174 For opsin negative control experiments, Black-6 mice were injected with virus encoding GFP

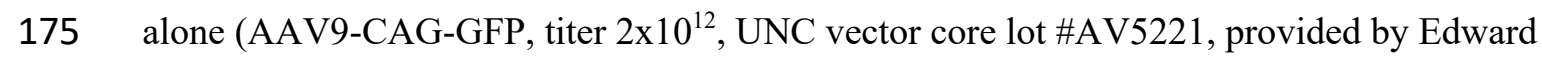
176 Boyden).

180 Procedures for epilepsy induction using the mouse unilateral intrahippocampal kainic acid model

181 of TLE largely followed previously published protocols (Cavalheiro et al., 1982; Bouilleret et al.,

182 1999; Bragin et al., 1999; Krook-Magnuson et al., 2013; Krook-Magnuson et al., 2014; Streng

183 and Krook-Magnuson, 2020b). A minimum of two weeks post viral injection(s), mice were

184 injected with $100 \mathrm{~nL}$ of kainic acid (KA) unilaterally into the right dorsal hippocampus (2.0 mm

185 posterior, $1.25 \mathrm{~mm}$ right, $1.6 \mathrm{~mm}$ ventral from bregma) under isoflurane anesthesia (2\%), as done

186 in our previous work (Streng and Krook-Magnuson, 2020b). Animals were removed from

187 isoflurane a maximum of five minutes post injection (Bar-Klein et al., 2016). In this model,

188 spontaneous recurrent electrographic seizures emerge from the damaged hippocampus, providing

189 a strong model of pharmocoresistant (Riban et al., 2002; Klein et al., 2015) temporal lobe

190 epilepsy with hippocampal sclerosis (Bouilleret et al., 1999; Riban et al., 2002; Levesque and 
191 Avoli, 2013; Zeidler et al., 2018). Only animals that showed spontaneous hippocampal seizures

192 weeks after KA injection, as determined by video EEG monitoring, were included for on-demand

193 optogenetic interventions.

\section{4}

\section{Electrode and fiber implantation}

196 A minimum of 1 week post kainic acid injection, mice were implanted with a twisted wire bipolar

197 (local reference, differential) electrode (PlasticsOne) ipsilateral to the site of kainate (2.6 mm

198 posterior, $1.75 \mathrm{~mm}$ right, $1.6 \mathrm{~mm}$ ventral from bregma). For fastigial output channel targeting

199 experiments, mice were additionally implanted with optical fibers targeting the left fastigial

200 nucleus (6.48 $\mathrm{mm}$ posterior, $0.75 \mathrm{~mm}$ left, $2.5 \mathrm{~mm}$ ventral from bregma). For fastigial terminal

201 targeting experiments, mice were implanted with optical fibers targeting the right central lateral

202 nucleus of the thalamus (1.34 $\mathrm{mm}$ posterior, $0.75 \mathrm{~mm}$ right, $2.75 \mathrm{~mm}$ ventral from bregma).

203 Implants were secured to the skull using screws and dental cement following previous protocols

204 (Armstrong et al., 2013; Krook-Magnuson et al., 2013) and allowed to recover a minimum of five

205 days prior to seizure monitoring and closed-loop interventions.

\section{Post-operative care}

208 For all surgical procedures, post-operative care consisted of recovery from anesthesia on a

209 heating pad with regular visual inspection, followed by daily post-operative monitoring for a

210 minimum of three days to inspect comfort level and healing of the surgical site. Neopredef

211 powder was applied to the closed incisions as a topical antibiotic and analgesic. In the case of

212 kainic acid injection, no additional post-operative analgesics were given. For viral injections and

213 implantations, carprofen was administered subcutaneously $(5 \mathrm{mg} / \mathrm{kg})$ immediately prior to

214 surgery. For implantations, pre- and post-operative ibuprofen was also administered orally (50-

$21580 \mathrm{mg} / \mathrm{kg} /$ day in water) as an additional analgesic. 


\section{Closed-loop seizure detection and interventions}

218 A minimum of five days post implantation, animals were placed in investigator managed housing

219 for chronic video and LFP recordings. Detection of electrographic seizures and on-demand

220 optogenetic interventions generally followed previously published protocols (Armstrong et al.,

221 2013; Krook-Magnuson et al., 2013; Streng and Krook-Magnuson, 2020b). Hippocampal LFP

222 was recorded via electrical patch cords through an electrical commutator (PlasticsOne), amplified

223 (Brownlee), digitized (National Instruments), and analyzed in real time by custom MATLAB

224 seizure detection software. A version of this software is available for download through

225 Armstrong et al, 2013 (Armstrong et al., 2013). Optogenetic interventions consisting of three

226 seconds of pulsed light delivery (50 msec on, $100 \mathrm{msec}$ off) (Streng and Krook-Magnuson,

227 2020b) were triggered for 50\% of events in a random fashion using this custom closed-loop

228 MATLAB software, and were delivered via LEDs $(473 \mathrm{~nm})$ through optical patch cords (Thor

229 labs). Stimulation parameters were selected due to their efficacy in robustly attenuating

230 hippocampal seizures when targeting the fastigial nucleus more broadly (Streng and Krook-

231 Magnuson, 2020b) or the cerebellar cortex (Krook-Magnuson et al., 2014), and were originally

232 selected to minimize movement side-effects (Krook-Magnuson et al., 2014). Post-hoc

233 measurements of light delivery indicated an average LED power of $1.5+/-0.6 \mathrm{~mW}$ at the tip of

234 the implanted optical fiber.

\section{Tissue harvesting and imaging of fibers}

237 In order to characterize viral expression and confirm appropriate optical fiber targeting, after

238 completion of on-demand interventions, mice were either deeply anesthetized with 5\% isoflurane

239 and decapitated or perfused with $0.1 \mathrm{M}$ phosphate buffer followed by $4 \%$ paraformaldehyde.

240 Brains were subsequently harvested and fixed in 4\% paraformaldehyde. Sagittal brain sections of

$24150 \mu \mathrm{m}$ were collected in $0.1 \mathrm{M}$ phosphate buffer using a vibratome (Leica VT1000S). After

242 sectioning, every third section was mounted with Vectashield mounting media with DAPI and 
243 covered with glass coverslips. Sagittal sections were initially visualized with epifluorescence

244 microscopy (Leica DM2500) to identify sections with structures of interest for confocal imaging.

245 The approximate coordinates of the sagittal slices and structures of interest were determined

246 using the Paxinos mouse brain atlas, third edition (Franklin and Paxinos, 2007) in combination

247 with DAPI and brightfield microscopy, which allowed for the discernment of areas of interest,

248 including thalamic nuclei. Confocal imaging was performed on an Olympus FluoView FV1000

249 BX2 upright confocal microscope (University Imaging Center, University of Minnesota), with

250 fields of view first selected using the DAPI signal to determine location (that is, blinded to the

251 specific eYFP fiber locations/densities). Medial lateral coordinates for the structures of interest

252 are as follows: $\mathrm{SC}(0.72 \mathrm{~mm}$ right $), \mathrm{MdV}(0.48 \mathrm{~mm}$ right $), \mathrm{CL}(0.72 \mathrm{~mm}$ right $)$, medial dorsal

253 nucleus, lateral part (MDL; $0.72 \mathrm{~mm}$ right), medial dorsal nucleus, central part (MDC; $0.48 \mathrm{~mm}$

254 right), ventral medial nucleus (VM, $0.72 \mathrm{~mm}$ right), ventral lateral nucleus (VL, $0.72 \mathrm{~mm}$ right),

255 parafascicular nucleus (PaF, $0.6 \mathrm{~mm}$ right), zona incerta (ZI, $1.44 \mathrm{~mm}$ right), mesencephalic

256 reticular formation ( $\mathrm{mRT}, 1.56 \mathrm{~mm}$ right), laterodorsal tegmental nucleus (LDTg, $0.48 \mathrm{~mm}$ right),

257 lateral periaqueductal grey (IPAG, $0.84 \mathrm{~mm}$ right), ventral lateral periaqueductal grey (vlPAG,

$2580.48 \mathrm{~mm}$ right), nucleus reticularis pontis caudalis (PnC, $0.48 \mathrm{~mm}$ right), parvocellular reticular

259 nucleus (PCRT, $1.20 \mathrm{~mm}$ right), and spinal vestibular nucleus (SpVe, $1.44 \mathrm{~mm}$ right).

260 Representative images were adjusted for brightness and contrast.

\section{Quantification of fibers}

263 After confocal images of eYFP labeled fibers in structures of interest were taken from FN-SC,

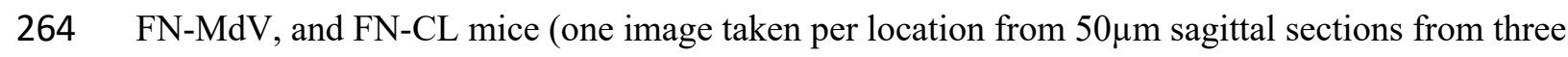

265 animals from each group), neurites within the imaged region of interest were measured using

266 Fiji's simple Neurite Tracer by tracing each neurite and the corresponding branches as separate

267 paths (note that quantification of fibers was done for only a subset of areas showing expression).

268 For the creation of a simplified summary schematic, the relative magnitude of fastigial fibers in a 
269 given location was assessed by comparing to the maximum observed in that area (for this, we

270 additionally quantified fibers in a mouse injected with virus for broad expression of ChR2-GFP).

271 Projections from a particular FN output channel to a region of interest were included in the

272 summary schematic if the average total path length was at least $15 \%$ of maximum and was

273 present at a minimum $15 \%$ level in at least two of the three animals quantified, with line thickness

274 proportional to the percent of maximum of the average total path length for that output location.

\section{Immunohistochemistry}

277 Our procedures for VGluT2 immunohistochemistry largely follow our previously published

278 protocol (Streng and Krook-Magnuson, 2020b). Briefly, every third sagittal section containing the

279 downstream target of interest was blocked with $10 \%$ bovine serum and $0.5 \%$ triton diluted in

280 TBS, followed by overnight incubation with primary antibody for VGluT2 at $4^{\circ} \mathrm{C}$ (Millipore

281 Sigma, 1:1000 diluted in TBS containing 2\% bovine serum and $0.4 \%$ triton). Tissue was then

282 rinsed and incubated for 2 hours with Alexa Fluor 594 anti-guinea pig (Jackson, 1:500 diluted in

283 TBS containing $2 \%$ bovine serum and $0.4 \%$ triton). Following secondary incubation, tissue was

284 mounted with Vectashield mounting media with DAPI and visualized with epifluorescence

285 microscopy (Leica DM2500) and confocal microscopy (Olympus FluoView FV1000 BX2) to

286 confirm colocalization with eYFP+ fibers.

288 For osteopontin (SPP1) immunohistochemistry, every third sagittal section of tissue containing

289 the left fastigial nucleus was blocked with $5 \%$ bovine serum albumin for one hour at room

290 temperature prior to incubation in primary antibody goat anti-osteopontin overnight at $4{ }^{\circ} \mathrm{C}$

291 (1:300, R\&D Systems AF808; RRID:AB_2194992). Tissue was then rinsed and incubated in

292 donkey anti-goat Alexa Fluor 594 for 2 hours at room temperature (1:500, ThermoFisher

293 Scientific A-11058). Following secondary incubation, tissue was mounted with Vectashield

294 mounting media with DAPI and viewed for colocalization of virally labeled and immunolabeled 
295 cell bodies using epifluorescence microscopy (Leica DM2500). Additional sections from an

296 additional animal (without immunohistochemistry) were also utilized for additional mapping of

297 virally-labeled somatic locations.

\section{Statistical analyses}

300 Seizure duration after the time of trigger and time to next seizure were analyzed off-line using a

301 combination of manual and automated methods consistent with our previous methods (Streng and

302 Krook-Magnuson, 2020b). Software for automated analysis is available for download through

303 github (https://github.com/KM-Lab/Electrographic-Seizure-Analyzer) (Zeidler et al., 2018). A

304 minimum of 100 seizure events per animal per condition were processed automatically based on

305 user-identified characteristics of spikes including amplitude, peak width, spike frequency and

306 deflection (positive, negative, or both), and the resulting post-detection seizure duration of all

307 events were confirmed via manual inspection. Post-detection seizure duration distributions

308 between light and no light conditions were compared in each animal using two-sample

309 Kolmogorov-Smirnov tests. To assess effectiveness of intervention at the group level, two

310 approaches were taken. First, a summative histogram of events was created, using an equal

311 number of events per animal, and the resulting distributions of light and no-light event durations

312 compared using two-sample Kolmogorov-Smirnov tests. Second, average post-detection seizure

313 durations (one value per condition per animal) were compared, and for visualization in figures,

314 post-detection seizure durations with light intervention were normalized by dividing by duration

315 without intervention (no light internal controls for the same animal), and expressed as a percent.

316 A $p$ value $<0.05$ was considered statistically significant. Statistical analyses were conducted

317 using MATLAB. Values are presented as mean $\pm \mathrm{SEM}$. 


\section{RESULTS}

\section{$321 \quad$ Fastigial outputs target numerous downstream structures}

322 On-demand excitation of the fastigial nucleus can robustly inhibit hippocampal seizures (Streng

323 and Krook-Magnuson, 2020b). However, how the fastigial nucleus can produce such a profound

324 influence on seizure activity is unclear. The fastigial projects to numerous downstream regions

325 (Angaut and Bowsher, 1970; Batton et al., 1977; Bentivoglio and Kuypers, 1982; Teune et al.,

326 2000; Fujita et al., 2020). We first visualized fastigial projections to potential downstream targets

327 of interest using a similar viral targeting strategy to our on-demand fastigial excitation work

328 (Streng and Krook-Magnuson, 2020b). Specifically, we injected Black-6 mice with an AAV to

329 achieve expression of the fluorescent protein GFP in neurons of the fastigial nucleus (AAV9-

330 CAG-GFP; Fig. 1A). With this approach, we labeled fastigial neurons in both rostral and caudal

331 portions of the fastigial nucleus (Fig. 1B-C), consistent with our previously published results

332 (Streng and Krook-Magnuson, 2020b).

334 Matching previous reports (Haroian et al., 1981; Asanuma et al., 1983; Angaut et al., 1985;

335 Gornati et al., 2018; Fujita et al., 2020), we found widespread axonal projection targets in

336 numerous regions (Fig. 1), including three areas of particular interest regarding potential seizure

337 suppression: the central lateral nucleus of the thalamus (CL, Fig 1Dii), superior colliculus (SC,

338 Fig. 1E), and medullary reticular formation (MdV, Fig 1Gii). As expected, fastigial terminals

339 were visible in multiple other thalamic nuclei (Fig 1D), including the ventral lateral (Fig. 1Diii),

340 parafascicular (Fig. 1Div), medial dorsal (Fig. 1Dv), ventral medial (Fig. 1Dvi), and zona incerta

341 nuclei (Fig. 1Dvii). In the midbrain, in addition to the superior colliculus (Fig. 1E), expression

342 was also observed in other mibrain nuclei (Fig. 1F) including the mesencephalic reticular

343 formation (Fig. 1Fii), laterodorsal tegmental nucleus (Fig. 1Fiii), and both lateral and ventral

344 lateral portions of the periaqueductal grey (Fig. 1Fiv-v). In the brainstem, fibers were observed in

345 multiple regions of the reticular formation (Fig. 1G, Gii, iv-vi) and vestibular nuclei (Fig. 1Giii). 
346 We also observed fastigial fibers traveling caudally through the medulla, likely comprising

347 cerebello-spinal tracts (Fig. 1Gv). Only a subset of regions with GFP+ terminals are presented

348 here; we observed a large number of projection targets, consistent with a recent report identifying

349 over 60 downstream targets (Fujita et al., 2020). The expression pattern after broad targeting of

350 the fastigial nucleus gave us a reference point for our next experiments looking at specific outputs

351 using a dual virus approach.

Fastigial outputs to the superior colliculus, medullary reticular formation, and central lateral

\section{4 nucleus comprise distinct populations}

355 To examine if neurons projecting to our three areas of interest (SC, MdV, and CL thalamus)

356 represented distinct neuronal populations, we utilized a dual viral strategy to achieve expression

357 of ChR2-eYFP in populations of fastigial neurons defined by their projection targets.

358 Specifically, mice were injected first in the downstream target of interest with an AAV designed

359 to provide retrograde expression of Cre (retroAAV-Cre) (Tervo et al., 2016), followed by

360 injection of the contralateral fastigial nucleus with a Cre-dependent virus for ChR2-eYFP

361 expression (Gradinaru et al., 2007). The result of these dual injections is ChR2-eYFP expression

362 only in fastigial neurons projecting to the downstream target of interest (Fig. 2A, B, C). We

363 injected retroAAV-Cre into either the SC (Fig. 2Aii), MdV (Fig. 2Bii), or CL thalamus (Fig.

364 2Cii), resulting in labeling of fastigial neurons that project to the $\mathrm{SC}, \mathrm{MdV}$, or CL, henceforth

365 referred to as FN-SC, FN-MdV, and FN-CL neurons, respectively.

367 FN-SC labeled neurons (Fig. 2Ai) were located in the caudal portion of the fastigial nucleus (Fig.

368 2Aiii, compare to Fig. 1B; Fig. 3D). As expected, FN-SC neurons had strong labeling of

369 terminals in the SC (Fig. 2Aiv, D,G). Importantly, fibers from FN-SC neurons were largely

370 absent from the MdV (Fig. 2H,K) and CL (Fig. 2L,O). Conversely, FN-MdV neurons (Fig. 2Bi)

371 were located throughout rostral and caudal portions of the fastigial nucleus (Fig. 2Biii, Fig. 3E), 
372 with some labeling extending into the interposed nucleus (Fig. 3E). Fibers from FN-MdV neurons

373 were strongly present in the MdV (Fig. 2Biv, I, K), but not SC (Fig. 2E, G) or CL (Fig. 2M, O).

374 Similar to FN-SC neurons, FN-CL neurons (Fig 2Ci) were preferentially located in the caudal

375 portion of the fastigial nucleus (Fig. 2Ciii, Fig. 3F). Similar independence of expression was

376 observed for FN-CL, with heavy labeling of terminals present in the CL (Fig. 2Civ, N-O), but not

377 SC (Fig. 2F-G) or MdV (Fig. 2J-K). This suggests that FN-SC, FN-MdV, and FN-CL cells

378 represent distinct populations of fastigial neurons. Note also that as all three populations included

379 at least some caudal labelling, full separation of these populations based solely on somatic

380 location would not be possible.

382 As an additional step, we characterized whether FN-SC, FN-MdV, or FN-CL neurons expressed

383 the molecular marker osteopontin (SPP1), which was recently characterized as a potential marker

384 for subpopulations of fastigial neurons (Fujita et al., 2020). We found both SPP1-immunopositive

$385\left(\mathrm{SPP}^{+}\right)$and SPP1-immunonegative (SPP1 ${ }^{-}$) fastigial neurons in all three populations (Fig. 3A-C).

386 However, the majority of neurons were SPP1-immunonegative, with only $19.6 \%$ (9/46 cells),

$38720.2 \%$ (19/143 cells), and 7.3\% (3/41 cells) immunopositive for SPP1 for FN-SC, FN-MDV, and

388 FN-CL neurons, respectively (Fig. 3D-F). While a smaller proportion of FN-CL cells were co-

389 labeled, compared to FN-SC or FN-MdV neurons, this was not statistically significant ( $\mathrm{p}=0.14$

390 and $\mathrm{p}=0.07$, respectively; Pearson's chi square). These results suggest that SPP1 is not a reliable

391 marker for FN-SC, FN-MDV, or FN-CL neurons.

393 Importantly, the location of fibers indicates that our dual labeling approach effectively labels

394 three distinct populations of fastigial neurons, in which fastigial neurons that project to the CL

395 nucleus do not project to the SC or MdV, and so on. This is predominantly in keeping with

396 recently reported findings (Fujita et al., 2020), and provided us with two important experimental

397 benefits. First, given that distinct populations of neurons were labeled, this approach provided a 
means to directly target FN-SC, FN-MdV, or FN-CL somata for circuit dissection of seizure

399 suppression, avoiding caveats associated with strategies optogenetically targeting terminals (e.g.,

400 insufficient light coverage). Additionally, this labeling approach allowed us to examine which (if

401 any) other downstream regions each of these populations project to, providing greater insight into

402 the circuit design of fastigial outputs.

404 We found that fastigial neurons that projected to the SC, MdV, or CL did, indeed, also project to

405 other areas, and that for many of these areas, segregation of these three output channels was

406 largely maintained (Figures 4-7).

408 Labeling of FN-SC neurons (Fig. 4A) also resulted in labeled fibers in several thalamic nuclei,

409 including the ventral lateral nucleus, and, to a lesser extent, the medial dorsal and ventral medial

410 nuclei (Fig. 4B-D). Some expression was also observed in the parafascicular nucleus in one

411 animal (Fig. 4E), and little to no observed in the zona incerta (Fig. 4F). In other regions of the

412 midbrain, expression was extremely limited (Fig. 4G-J). Similarly, essentially no fibers were

413 observed in the pontine reticular formation (Fig. 4K) or vestibular nuclei, and we saw no

414 evidence of FN-SC fibers travelling towards the spinal cord (Fig. 4L).

416 In contrast to FN-SC neurons, FN-MdV neurons (Fig. 5A) send relatively limited inputs to the

417 thalamus (Fig. 5B-F), with only the central portion of the medial dorsal nucleus showing dense

418 FN-MdV fibers (Fig. 5B). Within the midbrain (Fig. 5G-H), strong fiber labeling was seen in the

419 laterodorsal tegmental nucleus (Fig. 5H). The highest density of FN-MdV fibers was observed in

420 the brainstem, with extensive terminals in regions of the pontine (in addition to medullary)

421 reticular formation (Fig. 5I-J) and vestibular nuclei (Fig. 5K). This widespread brainstem

422 expression also included the gigantocellular reticular nucleus, the oral part of the pontine reticular

423 nucleus $(\mathrm{PnO})$, the medial vestibular nucleus and the superior vestibular nucleus. FN-MdV fibers 
424 in the brainstem largely appeared limited to local terminals, as we did not observe strong

425 evidence of FN-MdV fibers traveling to the spinal cord (Fig. 5L).

426

427 Labeling of FN-CL neurons (Fig. 6A) also resulted in labeling of fibers in additional thalamic

428 nuclei, including the medial dorsal (Fig. 6B-C) and ventral medial (Fig. 6D) thalamic nuclei, with

429 more limited projections to the parafascicular nucleus (Fig. 6F). Finally, FN-CL neurons also

430 appear to send very sparse projections to the zona incerta (Fig. 6G). As with FN-SC neurons, FN-

431 CL collaterals to the midbrain (Fig. 6H-K) and brainstem (Fig. 6L-M) were very limited. Finally,

432 similar to FN-SC and FN-MdV neurons, we did not observe evidence of FN-CL neurons sending

433 projections to the spinal cord (Fig. 6M).

435 Quantification of fibers from FN-SC, FN-MdV, and FN-CL neurons further supports that these

436 three populations send projections preferentially to largely segregated - but partially overlapping

437 - thalamic, midbrain, and brainstem nuclei (Fig. 7). Overall, of these three output channels, FN-

438 CL projecting neurons provided the strongest projections to the thalamus (Fig. 2O, Fig. 7A-F),

439 with the notable exception of the VL (Fig. 7D) which received more input from FN-SC neurons,

440 and the MDC (Fig. 7B), which received inputs from all three channels, but the heaviest from FN-

441 MdV neurons. In the midbrain, inputs from all three populations were largely limited and

442 somewhat overlapping (Fig. 7G-J), with FN-MdV inputs to the laterodorsal tegmental nucleus

443 being the most notable. While some fibers were found in the periaqueductal gray areas (Fig.

$4447 \mathrm{I}, \mathrm{J})$, these projections were extremely limited compared to the expression observed in animals

445 with broad fastigial targeting (Fig. 1F). Finally, fastigial inputs to the brainstem were massively

446 dominated by FN-MdV neurons, which send a high density of fibers throughout the medullary

447 (Fig. 2) and pontine reticular formation (Fig. 7K-L), as well as the vestibular nuclei (Fig. 7M).

448 Note that only a subset of targeted regions are illustrated in the figures and quantified in Figure 7. 
450 Together, these results indicate that there are distinct output channels from the fastigial nucleus,

451 and that FN-SC, FN-MdV, and FN-CL neurons comprise distinct populations, with different,

452 largely segregated, sets of target regions. FN-SC and FN-CL neurons each send projections to

453 unique thalamic nuclei, with more limited expression in the midbrain and brainstem (Fig. 7N, P).

454 Conversely, FN-MdV neurons send numerous projections to several nuclei within the brainstem

455 (Fig. 7O), and have extremely restricted projections to the thalamus. With this knowledge in

456 hand, we were able to test the impact of optogenetic manipulation of these populations of neurons

457 on seizures.

On-demand excitation of fastigial neurons projecting to the central lateral nucleus, but not SC

461 We utilized our dual viral strategy to implement on-demand optogenetic interventions in epileptic

462 mice, in order to determine whether fastigial influence over seizure activity can be achieved via

463 FN-SC, FN-MdV, or FN-CL neurons. Chronically epileptic animals were implanted with

464 recording electrodes in the hippocampus, and the local field potential signal was analyzed in real-

465 time to enable detection of spontaneous electrographic seizure activity and subsequent closed-

466 loop intervention (Armstrong et al., 2013). Light was delivered to the fastigial nucleus in animals

467 expressing channelrhodospin (ChR2) selectively in FN-SC, FN-MdV, or FN-CL neurons. Light

468 was delivered for half of detected seizure events, in a random fashion, allowing each animal to

469 serve as its own internal control.

471 Optogenetic excitation of FN-SC neurons (Fig. 8A) did not appear to have any effect on

472 hippocampal seizures (Fig. 8B-D). Across the population, optogenetic excitation of FN-SC

473 neurons failed to inhibit hippocampal seizures (Fig. 8D, p $=0.46$, Kolmogorov-Smirnov test.

474 Inset, $8 \pm 6 \%$ average reduction in seizure duration) with no significant effect of light observed in

475 any FN-SC animal tested (Fig. 8D inset, not significant in 6 of 6 animals). We also observed no 
476 effect on time to next seizure when modulating FN-SC neurons $(\mathrm{p}=0.31$, Wilcoxon signed rank

477 test, $\mathrm{n}=6$ animals).

478

479 Notably, because we targeted the somata of FN-SC neurons, we modulated the entire output

480 channel with this approach. This would suggest that these fastigial outputs to other areas targeted

481 by FN-SC neurons (e.g., ventral lateral thalamus) are also unlikely to contribute meaningfully to

482 the seizure suppressive effects of fastigial modulation. To further explore this possibility, we

483 utilized the same dual viral approach to target fastigial neurons traveling to the ventral lateral

484 nucleus of the thalamus (Fig. 8E-F), which project to both the ventral lateral nucleus (Fig. 8G,

485 left) and the superior colliculus (Fig. 8G, right). As with FN-SC modulation, on-demand

486 excitation of FN-VL neurons failed to attenuate hippocampal seizures (Fig. 8H-J), with no

487 significant effect of light delivery observed at the population (Fig. 8J, p = 0.48, Kolmogorov-

488 Smirnov test. Inset, average $8 \pm 13 \%$ increase in seizure duration) or individual animal level (Fig.

$4898 \mathrm{~J}$ inset, not significant in 4 out of 4 animals). Together, these results suggest that the FN-SC/FN-

490 VL outputs do not mediate the seizure suppression observed with broadly targeting FN output

491 neurons.

492

493 We next assessed whether excitation of FN-MdV neurons would have any effect on hippocampal

494 seizures (Fig. 9A). Similar to FN-SC neurons, optogenetic excitation of FN-MdV neurons did not

495 robustly attenuate hippocampal seizures (Fig. 9B-D). Across the population, a modest, albeit

496 statistically significant effect of light was observed (Fig, 9D, p = 0.046, Kolmogorov-Smirnov

497 test, Inset: average $5 \pm 17 \%$ reduction in post-detection seizure duration). However, at the

498 individual animal level, only 1 out of 6 animals showed a significant reduction in seizure duration

499 (Fig. 9D inset arrow; Fig.9E). In this one animal, the measured reduction in seizure duration

500 appeared to be more of a pause in seizure activity instead of a true attenuation (Fig 9F-H),

501 indicating that optogenetic excitation of FN-MdV neurons is insufficient to truly attenuate 
502 hippocampal seizures. As with FN-SC neurons, these results also suggest that other areas targeted

503 by FN-MdV neurons (e.g. vestibular nuclei, MDC, pontine reticular formation) are unlikely to be

504 driving fastigial control of hippocampal seizures.

506 In contrast to FN-SC and FN-MdV targeting, optogenetic excitation of FN-CL neurons was

507 sufficient to attenuate seizures (Fig. 10A-C). Across the population, targeting FN-CL neurons

508 robustly and consistently attenuated hippocampal seizures (Fig. 10D, p < 0.001, Kolmogorov-

509 Smirnov test) with all animals (7 of 7; Fig. 10D, Inset, average $34 \pm 17 \%$ reduction in post-

510 detection seizure duration) showing a significant effect of light delivery. This data indicates that

511 on-demand activation of fastigial neurons projecting to the CL nucleus of the thalamus is

512 sufficient for seizure inhibition. This finding is particularly striking given the lack of effect in

513 either FN-SC or FN-MdV animals; note that FN-MdV animals not only had extensive fibers to

514 numerous areas, but also had considerably more FN neurons labeled than FN-CL animals (Fig.

5153 ), indicating that this effect cannot be explained by relative expression levels.

517 In separate animals, we directly targeted the terminals of fastigial neurons in the CL (Fig. 10E-F).

518 Despite caveats mentioned above associated with targeting terminals, we found significant

519 inhibition of seizures with this approach (Fig. 10G, average $27 \pm 9 \%$ reduction, $p<0.001$

520 Kolmogorov-Smirnov test; inset: significant at the individual animal level in 7 of 11 animals).

521 This further supports that activation of FN-CL neurons is sufficient to attenuate hippocampal

522 seizures.

523

524 Confirming that the effect of light delivery to the CL was mediated via opsin-activation, no effect

525 was observed for opsin negative animals (injected with a virus for expression of GFP only)

526 receiving light to the CL (Fig. 10H). 
528 Across the populations, in contrast to effects seen in an FN-MdV animal, there was no effect on

529 time to next seizure when targeting FN-CL neurons $(p=0.297$, Wilcoxon signed rank test $)$, nor

530 when targeting fastigial terminals in the CL $(p=0.240$, Wilcoxon signed rank test $)$. At the

531 individual animal level, only one animal showed a significant effect of light delivery on time to

532 next seizure, but it was an increase in time to next seizure with light (rather than a decrease). This

533 suggests that FN-CL neurons produce a true seizure cessation (rather than brief pause).

534

535 Together, these results show that fastigial attenuation of hippocampal seizures can be achieved by

536 selectively exciting FN-CL neurons, but not by exciting FN-SC/FN-VL or FN-MdV neurons.

537 Our findings argue against the need for broad modulation of fastigial outputs, as activation of

538 fastigial outputs to the CL thalamus, selectively, is sufficient to inhibit seizures. More broadly,

539 our results highlight the concept of distinct fastigial output channels (Fig. 7N-P), and illustrate

540 that the existence of such distinct output channels can have important functional consequences

541 (Fig. 8-10).

542

543 


\section{DISCUSSION}

546 Using viral approaches coupled with neuroanatomical tracing and on-demand optogenetics, this

547 study reveals that a specific fastigial output channel is sufficient for attenuation of hippocampal

548 seizures. While the fastigial nucleus projects to a great many different target regions, we found

549 that outputs are organized into segregated channels. Specifically, fastigial neurons which project

550 to the SC also project heavily to the ventral lateral thalamus. Fastigial neurons projecting to the

551 MdV also target central regions of the medial dorsal thalamic nucleus, and send dense,

552 widespread, projections to brainstem reticular and vestibular nuclei. Finally, fastigial neurons that

553 project to the CL thalamus also project to the ventral medial and medial dorsal thalamic nuclei,

554 and to a lesser extent areas including zona incerta and the parafascicular nucleus. We found that

555 excitation of FN-CL neurons is sufficient to attenuate seizures, whereas excitation of fastigial

556 neurons projecting to the SC or MdV has no effect on seizures. Together, these results indicate

557 that fastigial control of hippocampal seizures does not require broad, concurrent manipulation of

558 several fastigial output channels; excitation of the fastigial output channel to CL thalamus is

559 sufficient. Notably, these findings also illustrate the functional relevance of distinct fastigial

560 output channels, and the potential usefulness of understanding cerebellar circuitry at this level.

562 We previously demonstrated that fastigial excitation can robustly attenuate hippocampal seizures

563 (Streng and Krook-Magnuson, 2020b). However, the lack of direct projections to the

564 hippocampus (Strick et al., 2009; Rochefort et al., 2013; Bohne et al., 2019; Watson et al., 2019;

565 Krook-Magnuson, 2020) requires that seizure cessation be mediated via connections with at least

566 one intermediate region. Despite being densely connected with a very large number of

567 downstream targets ( $>60$ total as estimated by Fujita et al 2020), we demonstrate that the fastigial

568 output channel to the CL nucleus of the thalamus is sufficient for seizure suppression. An

569 intralaminar nucleus considered to be part of the higher order thalamus (Saalmann, 2014), the CL

570 nucleus has been implicated in attention (Schiff et al., 2013), working memory (Wyder et al., 
571 2004), and arousal (Van der Werf et al., 2002). Especially relevant in the context of epilepsy and

572 seizures, it is hypothesized that the intralaminar thalamic nuclei serve to synchronize -- and

573 desynchronize -- the cerebral cortex in order to coordinate activity of cortical neurons and

574 networks (Saalmann, 2014). Focal limbic seizures modulate neuronal firing in the CL nucleus,

575 causing both an overall decrease in their activity levels and an increase in bursting (Feng et al.,

576 2017). Additionally, electrical stimulation of the CL nucleus during acutely evoked hippocampal

577 seizures can induce cortical desynchronization and improve measures of consciousness and

578 arousal (Gummadavelli et al., 2015; Kundishora et al., 2017; Xu et al., 2020), suggesting this

579 nucleus can play an active role in both participating in and influencing seizure networks. Our data

580 illustrate that perturbing activity in the CL nucleus may be able to inhibit seizures, as activation

581 of FN-CL neurons attenuated spontaneous hippocampal seizures (Fig. 10). Notably, we recorded

582 seizures in the hippocampus near the previous site of kainic acid injection and the presumed

583 seizure focus, likely indicating that the CL is ultimately able to impact "upstream" hippocampal

584 seizure activity. This may be through broad impacts on cortical synchrony (as noted above), or

585 specific effects e.g. on the anterior cingulate cortex (Van der Werf et al., 2002), which is reported

586 to have direct projections to the hippocampus (Rajasethupathy et al., 2015).

588 While our work utilized the intrahippocampal kainate mouse model of temporal lobe epilepsy, the 589 ability of the cerebellum to influence seizures may extend to those arising in regions outside the 590 hippocampus (reviewed in (Streng and Krook-Magnuson, 2020a)), including thalamocortical

591 absence seizures (Kros et al., 2015). It remains unknown whether cerebellar influence over

592 different seizure networks is also mediated by neurons projecting to the CL. In a recent study

593 examining thalamocortical absence seizures, activation of cerebellar nuclei terminals in the

594 thalamus was able to attenuate spike-and-wave discharges, but little appeared to be mediated via

595 the CL nucleus in particular (Eelkman Rooda et al., 2021). Indeed, that work tentatively

596 concluded that cerebellar inhibition of absence seizures was mediated via multiple nuclear output 
597 (channels) working together. This is in stark contrast to our findings; differences in outcomes

598 may be due to epilepsy type, cerebellar nucleus targeted, or experimental methods (i.e., we

599 primarily targeted somata within the cerebellar nucleus to avoid difficulties with selective and

600 sufficient terminal activation).

601

602 It is important to note that our experiments examine the FN-CL output channel, which has

603 collaterals to additional areas. Even for our fiber targeting experiments, in which light was

604 delivered directly to the CL, we cannot rule out potential antidromic activation. As such, other

605 areas FN-CL neurons project to, including the VM nucleus of the thalamus, may contribute to the

606 anti-seizure effects shown here. The VM projects to cortical areas including anterolateral motor

607 cortex (Guo et al., 2018) and infralimbic prefrontal cortex (Sieveritz and Arbuthnott, 2020), and

608 can target inhibitory neurons (and in particular, layer 1 neurogliaform cells) (Armstrong et al.,

609 2012; Overstreet-Wadiche and McBain, 2015; Anastasiades et al., 2021). Given the potential

610 interest of basal ganglia circuitry to seizures (Iadarola and Gale, 1982; Garant and Gale, 1987;

611 Wicker et al., 2019), it is also of interest that the VM receives inhibitory input from pars reticulata

612 (Kase et al., 2015). Additionally, neurons in this nucleus are highly modulated by spike-and-

613 wave discharges during absence seizures (Paz et al., 2007), and targeting cerebellar terminals in

614 the VM can provide some inhibition of spike-and-wave absence seizures (Eelkman Rooda et al.,

615 2021). However, the VM is also targeted by FN-SC neurons (Fig. 7N), albeit to a lesser extent,

616 and activation of FN-SC neurons did not inhibit hippocampal seizures (Fig. 8). Similarly, while

617 the MD is an additional target of FN-CL neurons and is of potential interest to seizure control

618 (Cassidy and Gale, 1998; Bertram et al., 2008; Sloan et al., 2011), the MD also receives input

619 from FN-SC and FN-MdV, which were ineffective in inhibiting seizures. Therefore, either the

$620 \mathrm{VM}$ and MD are playing little to no role in the seizure-inhibition effects of FN-CL neurons, FN-

$621 \mathrm{SC} / \mathrm{FN}-\mathrm{MdV}$ projections to the VM/MD are insufficient to achieve seizure inhibition, and/or FN-

$622 \mathrm{SC} / \mathrm{FN}-\mathrm{MdV}$ neurons and FN-CL neurons project to different populations of VM/MD neurons. 
624 It is important to note that our results firmly show that not all thalamic nuclei which receive

625 fastigial input provide seizure inhibition effects. Specifically, we show that activation of FN-SC

626 neurons, which have collaterals to the VL thalamus, does not inhibit seizures. We additionally

627 directly targeted FN-VL neurons, and again saw no inhibition of seizures. Therefore, fastigial

628 inhibition of seizures is not mediated by fastigial-thalamic projections in a generic sense, but

629 rather by FN-CL neurons specifically.

631 In this regard, in addition to identifying key fastigial outputs necessary for seizure cessation, our

632 results provide important evidence as to which targets may not be involved in mediating the anti-

633 seizure effects of direct fastigial modulation. Given that direct optogenetic modulation of the SC

634 is highly effective in disrupting seizures in several rodent models of different types of epilepsy

635 (Soper et al., 2016), we initially reasoned that fastigial projections to the SC might be mediating

636 fastigial inhibition of seizures. However, somewhat surprisingly, on-demand optogenetic

637 excitation of FN-SC neurons failed to inhibit hippocampal seizures. Previous work has examined

638 SC influence on seizures not arising in the hippocampus per se (Iadarola and Gale, 1982; Gale et

639 al., 1993; Soper et al., 2016). As such, one possibility is that the SC lacks the ability to influence

640 hippocampal seizures specifically. Alternatively, as with FN-SC projections to the VL or MD

641 thalamus, FN projections to the SC may target the wrong population of SC neurons (e.g., ones

642 that do not mediate seizure suppression), or may simply provide insufficiently strong input to the

643 SC. Regardless, fastigial control of hippocampal seizures does not appear to be mediated via the

644 SC.

646 We also show that excitation of fastigial projections to the medullary reticular formation fails to

647 attenuate seizures. This argues against the hypothesis that seizure suppression is mediated by a

648 reticular-formation-induced brain state change (Pfaff et al., 2012; Ewell et al., 2015; Khan et al., 
649 2018; Purnell et al., 2018; Sakai, 2018; Streng and Krook-Magnuson, 2020a). Given the extensive

650 fibers observed with FN-MdV labeling, as well as the distribution of cell bodies throughout the

651 fastigial nucleus, the FN-MdV output channel may actually be comprised of multiple sub-

652 channels. These sub-channels may contain different aggregates of additional downstream targets,

653 such as those described previously (Teune et al., 2000). None appear to mediate seizure

654 inhibition. Our results additionally indicate that other regions receiving strong input from FN-

$655 \mathrm{MdV}$ neurons, including the central portion of the MD thalamus or the vestibular nuclei, are

656 unlikely to be significantly contributing to suppression of hippocampal seizures.

658 While we cannot rule out that other FN output channels not tested in this study may also be able

659 to inhibit seizures, the robust effect of FN-CL modulation, coupled with the lack of an effect

660 when modulating either FN-SC or FN-MdV output channels, strongly argues against suppression

661 of hippocampal seizures requiring coordinated changes across a large number of fastigial targets.

662 Rather, there are particular pathway(s) - starting with the FN-CL output pathway -- by which the

663 cerebellum influences the hippocampus and hippocampal seizures. While a different pathway

664 and/or output channel may be at play in healthy animals, it is worth noting that temporally precise

665 modulation of hippocampal activity is observed with stimulation of cerebellar neurons in non-

666 epileptic animals (Choe et al., 2018; Zeidler et al., 2020). Additionally, multisynaptic rabies

667 tracing, following injections into the hippocampal dentate gyrus (again, in non-epileptic animals),

668 results in labeling of neurons in the fastigial nucleus (Watson et al., 2019), futher supporting a

669 (multisynaptic) connection between these structures. Notably, this rabies labeling is seen in the

670 caudal portion of the fastigial nucleus -- the same region in which we observe FN-CL neurons

671 (Fig. 2Ciii), suggesting that hippocampal activity is influenced by this subpopulation of fastigial

672 neurons.

673 
674 The fastigial nucleus, considered part of the functional division of the cerebellum known as the

675 spinocerebellum, has canonically been considered to be mostly involved in motor functions of

676 proximal muscles and eye movements (Purves et al., 2001). While early work (Angaut and

677 Bowsher, 1970; Batton et al., 1977; Bentivoglio and Kuypers, 1982; Angaut et al., 1985)

678 has characterized some of the projection targets of cerebellar nuclei, including several thalamic

679 and brainstem nuclei, and suggested some segregation of outputs based on somatic location

680 within the fastigial nucleus (Bentivoglio and Kuypers, 1982; Noda et al., 1990; Fuchs et al., 1993;

681 Teune et al., 2000), we are just beginning to appreciate the full diversity and complexity of

682 fastigial projections to both motor and non-motor structures. A recent study examined output

683 from fastigial neurons by subregion within the fastigial nucleus, and suggested 5 distinct output

684 channels (Fujita et al., 2020), 3 of which bear many similarities with the populations we

685 characterize here. In that study by Fujita and colleagues, one population of neurons in the caudal

686 fastigial nucleus (and to some extent the caudal dorsolateral protuberance) projected to areas

687 including the CL, MD, and VM thalamus (similar to our FN-CL channel). A second population

688 of neurons in the caudal dorsolateral protuberance (and to some extent caudal fastigial) projected

689 to areas including the VL and PaF thalamus, zona incerta, and SC (somewhat in keeping with our

690 FN-SC channel). A third population of neurons in more rostral portions of the fastigial projected

691 to areas including the medullary reticular formation, vestibular nuclei, and spinal cord (apparently

692 encompassing some of our FN-MdV channel and additional channel(s)). In total, these

693 experiments provide a wealth of insights into fastigial outputs. However, the experimental

694 approaches taken did not allow segregation of output channels beyond immunohistochemical

695 profile or general location within the nucleus - for example, it was not clear from those results if

696 the same neuron projects to a single downstream area, to multiple downstream areas, or to all

697 downstream areas identified as receiving input from a given region of the fastigial nucleus.

698 Therefore, while Fujita et al identified a region of fastigial neurons which contains projections to

699 the CL, ventral medial, and medial dorsal nuclei, we specifically show that fastigial neurons that 
700 project to the CL have collaterals to the ventral medial and medial dorsal thalamus - that is, the

701 same neuron projects to multiple regions. Given the partial blurring of anatomical regions within

702 the fastigial seen by Fujita and colleagues, it is also difficult to know from those studies if the

703 output channels identified were non-overlapping. Indeed, our work suggests that while the FN-

704 CL, FN-SC, and FN-MdV populations are distinct, there are areas of overlap which receive input

705 from multiple output channels (e.g. the medial dorsal nucleus). We also note some additional

706 slight differences in the inferred organization of outputs. Our results thus illustrate a high level of

707 nuance in fastigial outputs, with an increased number of output channels than the five which were

708 recently suggested, and where specific fastigial output channels send collateral projections to both

709 convergent and divergent downstream targets.

710

711 The existence of individual fastigial neurons that project simultaneously to multiple, distinct,

712 downstream regions is intriguing, as is their functional significance pertaining to ongoing,

713 healthy, cerebellar function. One potential framework for understanding these simultaneous

714 projections is the forward-internal-model hypothesis of cerebellar function, in which the

715 cerebellum generates predictions about the sensory consequences of actions (Wolpert et al., 1998;

716 Kawato, 1999; Popa and Ebner, 2018). These predictions of sensory consequences are used both

717 to influence and correct ongoing processes as well as optimize future actions (for review, see

718 (Popa et al., 2016)); fastigial collaterals could convey predictions to regions that would update

719 ongoing motor commands and the motor controller. The existence of segregated channels may

720 also be compatible with a multiple forward internal model framework; the cerebellum may

721 implement forward internal models in motor and non-motor domains (Popa et al., 2014;

722 Diedrichsen et al., 2019), via separate output channels. Alternatively, different regions and

723 modules of the cerebellum may implement distinct functional roles (Diedrichsen et al., 2019). In

724 either framework, FN-CL neurons may support more cognitive functions. 
726 Together, our results shed important light on the necessary outputs for fastigial control of

727 hippocampal seizures and demonstrate the complexity of fastigial outputs and projections. Rather

728 than requiring broad modulation of fastigial outputs, we show that seizure suppression is

729 achievable via FN-CL neurons, outlining new potential avenues for selective therapeutic

730 interventions with minimized side effects. Our results also underscore the sophistication of the

731 fastigial nucleus, whereby specific fastigial output channels, which project to specific

732 downstream targets, carry distinct functional outcomes.

733 


\section{Funding}

736

737 This work was supported in part by The Winston and Maxine Wallin Neuroscience Discovery

738 Fund Award, an American Epilepsy Society Postdoctoral Fellowship (MLS), NIH R01-

739 NS112518, a University of Minnesota McKnight Land-Grant Professorship award, and the

740 University of Minnesota’s MnDRIVE (Minnesota’s Discovery, Research and Innovation

741 Economy) initiative. 


\section{References}

744 Anastasiades PG, Collins DP, Carter AG (2021) Mediodorsal and Ventromedial Thalamus Engage Distinct L1 Circuits in the Prefrontal Cortex. Neuron 109:314330 e314.

Andersen BB, Korbo L, Pakkenberg B (1992) A quantitative study of the human cerebellum with unbiased stereological techniques. J Comp Neurol 326:549-560.

Andrezik JA, Dormer KJ, Foreman RD, Person RJ (1984) Fastigial nucleus projections to the brain stem in beagles: pathways for autonomic regulation. Neuroscience 11:497-507.

Angaut P, Bowsher D (1970) Ascending projections of the medial cerebellar (fastigial) nucleus: an experimental study in the cat. Brain Res 24:49-68.

Angaut P, Cicirata F, Serapide F (1985) Topographic organization of the cerebellothalamic projections in the rat. An autoradiographic study. Neuroscience 15:389-401.

Armstrong C, Krook-Magnuson E, Soltesz I (2012) Neurogliaform and Ivy Cells: A Major Family of nNOS Expressing GABAergic Neurons. Front Neural Circuits 6:23.

Armstrong C, Krook-Magnuson E, Oijala M, Soltesz I (2013) Closed-loop optogenetic intervention in mice. Nat Protoc 8:1475-1493.

Asanuma C, Thach WT, Jones EG (1983) Cytoarchitectonic delineation of the ventral lateral thalamic region in the monkey. Brain Res 286:219-235.

Bar-Klein G, Klee R, Brandt C, Bankstahl M, Bascunana P, Tollner K, Dalipaj H, Bankstahl JP, Friedman A, Loscher W (2016) Isoflurane prevents acquired epilepsy in rat models of temporal lobe epilepsy. Ann Neurol 80:896-908.

Batton RR, 3rd, Jayaraman A, Ruggiero D, Carpenter MB (1977) Fastigial efferent projections in the monkey: an autoradiographic study. J Comp Neurol 174:281305.

Bentivoglio M, Kuypers HG (1982) Divergent axon collaterals from rat cerebellar nuclei to diencephalon, mesencephalon, medulla oblongata and cervical cord. A fluorescent double retrograde labeling study. Exp Brain Res 46:339-356.

Bertram EH, Zhang D, Williamson JM (2008) Multiple roles of midline dorsal thalamic nuclei in induction and spread of limbic seizures. Epilepsia 49:256-268.

Bohne P, Schwarz MK, Herlitze S, Mark MD (2019) A New Projection From the Deep Cerebellar Nuclei to the Hippocampus via the Ventrolateral and Laterodorsal Thalamus in Mice. Front Neural Circuits 13:51.

Bouilleret V, Ridoux V, Depaulis A, Marescaux C, Nehlig A, Le Gal La Salle G (1999) Recurrent seizures and hippocampal sclerosis following intrahippocampal kainate injection in adult mice: electroencephalography, histopathology and synaptic reorganization similar to mesial temporal lobe epilepsy. Neuroscience 89:717729.

Bragin A, Engel J, Jr., Wilson CL, Vizentin E, Mathern GW (1999) Electrophysiologic analysis of a chronic seizure model after unilateral hippocampal KA injection. Epilepsia 40:1210-1221.

Cassidy RM, Gale K (1998) Mediodorsal thalamus plays a critical role in the development of limbic motor seizures. J Neurosci 18:9002-9009. 
Cavalheiro EA, Riche DA, Le Gal La Salle G (1982) Long-term effects of intrahippocampal kainic acid injection in rats: a method for inducing spontaneous recurrent seizures. Electroencephalogr Clin Neurophysiol 53:581-589.

Choe KY, Sanchez CF, Harris NG, Otis TS, Mathews PJ (2018) Optogenetic fMRI and electrophysiological identification of region-specific connectivity between the cerebellar cortex and forebrain. Neuroimage 173:370-383.

Chung K, Wallace J, Kim SY, Kalyanasundaram S, Andalman AS, Davidson TJ, Mirzabekov JJ, Zalocusky KA, Mattis J, Denisin AK, Pak S, Bernstein H, Ramakrishnan C, Grosenick L, Gradinaru V, Deisseroth K (2013) Structural and molecular interrogation of intact biological systems. Nature 497:332-337.

Colombel C, Lalonde R, Caston J (2004) The effects of unilateral removal of the cerebellar hemispheres on spatial learning and memory in rats. Brain Res 1004:108-115.

Dean P, Gale K (1989) Anticonvulsant action of GABA receptor blockade in the nigrotectal target region. Brain Res 477:391-395.

Diedrichsen J, King M, Hernandez-Castillo C, Sereno M, Ivry RB (2019) Universal Transform or Multiple Functionality? Understanding the Contribution of the Human Cerebellum across Task Domains. Neuron 102:918-928.

Eelkman Rooda OHJ, Kros L, Faneyte SJ, Holland PJ, Gornati SV, Poelman HJ, Jansen NA, Tolner EA, van den Maagdenberg A, De Zeeuw CI, Hoebeek FE (2021) Single-pulse stimulation of cerebellar nuclei stops epileptic thalamic activity. Brain Stimul 14:861-872.

England MJ, Liverman CT, Schultz AM, Strawbridge LM (2012) Epilepsy across the spectrum: promoting health and understanding. A summary of the Institute of Medicine report. Epilepsy Behav 25:266-276.

Ewell LA, Liang L, Armstrong C, Soltesz I, Leutgeb S, Leutgeb JK (2015) Brain State Is a Major Factor in Preseizure Hippocampal Network Activity and Influences Success of Seizure Intervention. J Neurosci 35:15635-15648.

Feng L, Motelow JE, Ma C, Biche W, McCafferty C, Smith N, Liu M, Zhan Q, Jia R, Xiao B, Duque A, Blumenfeld H (2017) Seizures and Sleep in the Thalamus: Focal Limbic Seizures Show Divergent Activity Patterns in Different Thalamic Nuclei. J Neurosci 37:11441-11454.

Fenno LE, Mattis J, Ramakrishnan C, Hyun M, Lee SY, He M, Tucciarone J, Selimbeyoglu A, Berndt A, Grosenick L, Zalocusky KA, Bernstein H, Swanson H, Perry C, Diester I, Boyce FM, Bass CE, Neve R, Huang ZJ, Deisseroth K (2014) Targeting cells with single vectors using multiple-feature Boolean logic. Nat Methods 11:763-772.

Franklin K, Paxinos G (2007) The mouse brain in stereotaxic coordinates, Third Edition. New York, NY: Elsevier.

Fuchs AF, Robinson FR, Straube A (1993) Role of the caudal fastigial nucleus in saccade generation. I. Neuronal discharge pattern. J Neurophysiol 70:1723-1740.

Fujita H, Kodama T, du Lac S (2020) Modular output circuits of the fastigial nucleus for diverse motor and nonmotor functions of the cerebellar vermis. Elife 9.

Gale K, Pazos A, Maggio R, Japikse K, Pritchard P (1993) Blockade of GABA receptors in superior colliculus protects against focally evoked limbic motor seizures. Brain Res 603:279-283. 
834 Garant DS, Gale K (1987) Substantia nigra-mediated anticonvulsant actions: role of nigral output pathways. Exp Neurol 97:143-159.

Gornati SV, Schafer CB, Eelkman Rooda OHJ, Nigg AL, De Zeeuw CI, Hoebeek FE (2018) Differentiating Cerebellar Impact on Thalamic Nuclei. Cell Rep 23:26902704.

Gradinaru V, Thompson KR, Zhang F, Mogri M, Kay K, Schneider MB, Deisseroth K (2007) Targeting and readout strategies for fast optical neural control in vitro and in vivo. J Neurosci 27:14231-14238.

Gummadavelli A, Motelow JE, Smith N, Zhan Q, Schiff ND, Blumenfeld H (2015) Thalamic stimulation to improve level of consciousness after seizures: evaluation of electrophysiology and behavior. Epilepsia 56:114-124.

Guo K, Yamawaki N, Svoboda K, Shepherd GMG (2018) Anterolateral Motor Cortex Connects with a Medial Subdivision of Ventromedial Thalamus through Cell Type-Specific Circuits, Forming an Excitatory Thalamo-Cortico-Thalamic Loop via Layer 1 Apical Tuft Dendrites of Layer 5B Pyramidal Tract Type Neurons. J Neurosci 38:8787-8797.

Haroian AJ, Massopust LC, Young PA (1981) Cerebellothalamic projections in the rat: an autoradiographic and degeneration study. J Comp Neurol 197:217-236.

Hilber P, Jouen F, Delhaye-Bouchaud N, Mariani J, Caston J (1998) Differential roles of cerebellar cortex and deep cerebellar nuclei in learning and retention of a spatial task: studies in intact and cerebellectomized lurcher mutant mice. Behav Genet 28:299-308.

Howarth C, Peppiatt-Wildman CM, Attwell D (2010) The energy use associated with neural computation in the cerebellum. J Cereb Blood Flow Metab 30:403-414.

Iadarola MJ, Gale K (1982) Substantia nigra: site of anticonvulsant activity mediated by gamma-aminobutyric acid. Science 218:1237-1240.

Jones BE (2003) Arousal systems. Front Biosci 8:s438-451.

Kase D, Uta D, Ishihara H, Imoto K (2015) Inhibitory synaptic transmission from the substantia nigra pars reticulata to the ventral medial thalamus in mice. Neurosci Res 97:26-35.

Kawato M (1999) Internal models for motor control and trajectory planning. Curr Opin Neurobiol 9:718-727.

Khan S, Nobili L, Khatami R, Loddenkemper T, Cajochen C, Dijk DJ, Eriksson SH (2018) Circadian rhythm and epilepsy. Lancet Neurol 17:1098-1108.

Kipping JA, Grodd W, Kumar V, Taubert M, Villringer A, Margulies DS (2013) Overlapping and parallel cerebello-cerebral networks contributing to sensorimotor control: an intrinsic functional connectivity study. Neuroimage 83:837-848.

Klein S, Bankstahl M, Loscher W (2015) Inter-individual variation in the effect of antiepileptic drugs in the intrahippocampal kainate model of mesial temporal lobe epilepsy in mice. Neuropharmacology 90:53-62.

Krook-Magnuson E (2020) From Point A to Point B, and What it Means for Epilepsy. Epilepsy Curr 20:51-53.

Krook-Magnuson E, Armstrong C, Oijala M, Soltesz I (2013) On-demand optogenetic control of spontaneous seizures in temporal lobe epilepsy. Nat Commun 4:1376. 
878 Krook-Magnuson E, Szabo GG, Armstrong C, Oijala M, Soltesz I (2014) Cerebellar Directed Optogenetic Intervention Inhibits Spontaneous Hippocampal Seizures in a Mouse Model of Temporal Lobe Epilepsy. eNeuro 1.

Kros L, Eelkman Rooda OH, Spanke JK, Alva P, van Dongen MN, Karapatis A, Tolner EA, Strydis C, Davey N, Winkelman BH, Negrello M, Serdijn WA, Steuber V, van den Maagdenberg AM, De Zeeuw CI, Hoebeek FE (2015) Cerebellar output controls generalized spike-and-wave discharge occurrence. Ann Neurol 77:10271049.

Kundishora AJ, Gummadavelli A, Ma C, Liu M, McCafferty C, Schiff ND, Willie JT, Gross RE, Gerrard J, Blumenfeld H (2017) Restoring Conscious Arousal During Focal Limbic Seizures with Deep Brain Stimulation. Cereb Cortex 27:1964-1975.

Lefort JM, Vincent J, Tallot L, Jarlier F, De Zeeuw CI, Rondi-Reig L, Rochefort C (2019) Impaired cerebellar Purkinje cell potentiation generates unstable spatial map orientation and inaccurate navigation. Nat Commun 10:2251.

Leggio MG, Neri P, Graziano A, Mandolesi L, Molinari M, Petrosini L (1999) Cerebellar contribution to spatial event processing: characterization of procedural learning. Exp Brain Res 127:1-11.

Levesque M, Avoli M (2013) The kainic acid model of temporal lobe epilepsy. Neurosci Biobehav Rev 37:2887-2899.

Middleton FA, Strick PL (1998) Cerebellar output: motor and cognitive channels. Trends Cogn Sci 2:348-354.

Moruzzi G, Magoun HW (1949) Brain stem reticular formation and activation of the EEG. Electroencephalogr Clin Neurophysiol 1:455-473.

Noda H, Sugita S, Ikeda Y (1990) Afferent and efferent connections of the oculomotor region of the fastigial nucleus in the macaque monkey. J Comp Neurol 302:330348.

Overstreet-Wadiche L, McBain CJ (2015) Neurogliaform cells in cortical circuits. Nat Rev Neurosci 16:458-468.

Paz JT, Chavez M, Saillet S, Deniau JM, Charpier S (2007) Activity of ventral medial thalamic neurons during absence seizures and modulation of cortical paroxysms by the nigrothalamic pathway. J Neurosci 27:929-941.

Pfaff DW, Martin EM, Faber D (2012) Origins of arousal: roles for medullary reticular neurons. Trends Neurosci 35:468-476.

Popa LS, Ebner TJ (2018) Cerebellum, Predictions and Errors. Front Cell Neurosci 12:524.

Popa LS, Hewitt AL, Ebner TJ (2014) The cerebellum for jocks and nerds alike. Front Syst Neurosci 8:113.

Popa LS, Streng ML, Hewitt AL, Ebner TJ (2016) The Errors of Our Ways: Understanding Error Representations in Cerebellar-Dependent Motor Learning. Cerebellum 15:93-103.

Purnell BS, Thijs RD, Buchanan GF (2018) Dead in the Night: Sleep-Wake and TimeOf-Day Influences on Sudden Unexpected Death in Epilepsy. Front Neurol 9:1079.

Purves D, Augustine GJ, Fitzpatrick D, Hall WC, LaMantia A-S, McNamara JO, \& Williams SM (2001) Neuroscience (3rd ed). Sunderland, MA: Sinauer Associates. 
923 Rajasethupathy P, Sankaran S, Marshel JH, Kim CK, Ferenczi E, Lee SY, Berndt A, Ramakrishnan C, Jaffe A, Lo M, Liston C, Deisseroth K (2015) Projections from neocortex mediate top-down control of memory retrieval. Nature 526:653-659.

Ramnani N (2006) The primate cortico-cerebellar system: anatomy and function. Nat Rev Neurosci 7:511-522.

Ramnani N (2012) Frontal lobe and posterior parietal contributions to the corticocerebellar system. Cerebellum 11:366-383.

Riban V, Bouilleret V, Pham-Le BT, Fritschy JM, Marescaux C, Depaulis A (2002) Evolution of hippocampal epileptic activity during the development of hippocampal sclerosis in a mouse model of temporal lobe epilepsy. Neuroscience 112:101-111.

Rochefort C, Lefort JM, Rondi-Reig L (2013) The cerebellum: a new key structure in the navigation system. Front Neural Circuits 7:35.

Rochefort C, Arabo A, Andre M, Poucet B, Save E, Rondi-Reig L (2011) Cerebellum shapes hippocampal spatial code. Science 334:385-389.

Roldan M, Reinoso-Suarez F (1981) Cerebellar projections to the superior colliculus in the cat. J Neurosci 1:827-834.

Saalmann YB (2014) Intralaminar and medial thalamic influence on cortical synchrony, information transmission and cognition. Front Syst Neurosci 8:83.

Sakai K (2018) Behavioural state-specific neurons in the mouse medulla involved in sleep-wake switching. Eur J Neurosci 47:1482-1503.

Salanova V et al. (2015) Long-term efficacy and safety of thalamic stimulation for drugresistant partial epilepsy. Neurology 84:1017-1025.

Salmi J, Pallesen KJ, Neuvonen T, Brattico E, Korvenoja A, Salonen O, Carlson S (2010) Cognitive and motor loops of the human cerebro-cerebellar system. J Cogn Neurosci 22:2663-2676.

Schiff ND, Shah SA, Hudson AE, Nauvel T, Kalik SF, Purpura KP (2013) Gating of attentional effort through the central thalamus. J Neurophysiol 109:1152-1163.

Schmahmann JD (1996) From movement to thought: anatomic substrates of the cerebellar contribution to cognitive processing. Hum Brain Mapp 4:174-198.

Schmahmann JD (2019) The cerebellum and cognition. Neurosci Lett 688:62-75.

Shipman ML, Green JT (2019) Cerebellum and cognition: Does the rodent cerebellum participate in cognitive functions? Neurobiol Learn Mem:106996.

Sieveritz B, Arbuthnott GW (2020) Prelimbic cortical targets of ventromedial thalamic projections include inhibitory interneurons and corticostriatal pyramidal neurons in the rat. Brain Struct Funct 225:2057-2076.

Sloan DM, Zhang D, Bertram EH, 3rd (2011) Increased GABAergic inhibition in the midline thalamus affects signaling and seizure spread in the hippocampusprefrontal cortex pathway. Epilepsia 52:523-530.

Soper C, Wicker E, Kulick CV, N'Gouemo P, Forcelli PA (2016) Optogenetic activation of superior colliculus neurons suppresses seizures originating in diverse brain networks. Neurobiol Dis 87:102-115.

Streng ML, Krook-Magnuson E (2020a) The cerebellum and epilepsy. Epilepsy Behav:106909. 
1004

1005

1006

1007

1008

1009

1010

Streng ML, Krook-Magnuson E (2020b) Excitation, but not inhibition, of the fastigial nucleus provides powerful control over temporal lobe seizures. J Physiol 598:171187.

Strick PL, Dum RP, Fiez JA (2009) Cerebellum and nonmotor function. Annu Rev Neurosci 32:413-434.

Tervo DG, Hwang BY, Viswanathan S, Gaj T, Lavzin M, Ritola KD, Lindo S, Michael S, Kuleshova E, Ojala D, Huang CC, Gerfen CR, Schiller J, Dudman JT, Hantman AW, Looger LL, Schaffer DV, Karpova AY (2016) A Designer AAV Variant Permits Efficient Retrograde Access to Projection Neurons. Neuron 92:372-382.

Teune TM, van der Burg J, van der Moer J, Voogd J, Ruigrok TJ (2000) Topography of cerebellar nuclear projections to the brain stem in the rat. Prog Brain Res 124:141-172.

Van der Werf YD, Witter MP, Groenewegen HJ (2002) The intralaminar and midline nuclei of the thalamus. Anatomical and functional evidence for participation in processes of arousal and awareness. Brain Res Brain Res Rev 39:107-140.

Vong L, Ye C, Yang Z, Choi B, Chua S, Jr., Lowell BB (2011) Leptin action on GABAergic neurons prevents obesity and reduces inhibitory tone to POMC neurons. Neuron 71:142-154.

Watson TC, Obiang P, Torres-Herraez A, Watilliaux A, Coulon P, Rochefort C, RondiReig L (2019) Anatomical and physiological foundations of cerebellohippocampal interaction. Elife 8.

Weng X, Rosenberg HC (1992) Infusion of bicuculline methiodide into the tectum: model specificity of pro- and anticonvulsant actions. Epilepsy Res 12:1-8.

Wicker E, Forcelli PA (2016) Chemogenetic silencing of the midline and intralaminar thalamus blocks amygdala-kindled seizures. Exp Neurol 283:404-412.

Wicker E, Beck VC, Kulick-Soper C, Kulick-Soper CV, Hyder SK, Campos-Rodriguez C, Khan T, N'Gouemo P, Forcelli PA (2019) Descending projections from the substantia nigra pars reticulata differentially control seizures. Proc Natl Acad Sci U S A.

Wolpert DM, Miall RC, Kawato M (1998) Internal models in the cerebellum. Trends Cogn Sci 2:338-347.

Wyder MT, Massoglia DP, Stanford TR (2004) Contextual modulation of central thalamic delay-period activity: representation of visual and saccadic goals. J Neurophysiol 91:2628-2648.

Xu J, Galardi MM, Pok B, Patel KK, Zhao CW, Andrews JP, Singla S, McCafferty CP, Feng L, Musonza ET, Kundishora AJ, Gummadavelli A, Gerrard JL, Laubach M, Schiff ND, Blumenfeld H (2020) Thalamic Stimulation Improves Postictal Cortical Arousal and Behavior. J Neurosci 40:7343-7354.

Yu W, Krook-Magnuson E (2015) Cognitive Collaborations: Bidirectional Functional Connectivity Between the Cerebellum and the Hippocampus. Front Syst Neurosci 9:177.

Zeidler Z, Hoffman K, Krook-Magnuson E (2020) HippoBellum: acute cerebellar modulation alters hippocampal dynamics and function. In press. 
1011 Zeidler Z, Brandt-Fontaine M, Leintz C, Krook-Magnuson C, Netoff T, Krook-

$1012 \quad$ Magnuson E (2018) Targeting the Mouse Ventral Hippocampus in the

1013 Intrahippocampal Kainic Acid Model of Temporal Lobe Epilepsy. eNeuro 5.

1014 Zhang XY, Wang JJ, Zhu JN (2016) Cerebellar fastigial nucleus: from anatomic

1015

1016 construction to physiological functions. Cerebellum Ataxias 3:9. 

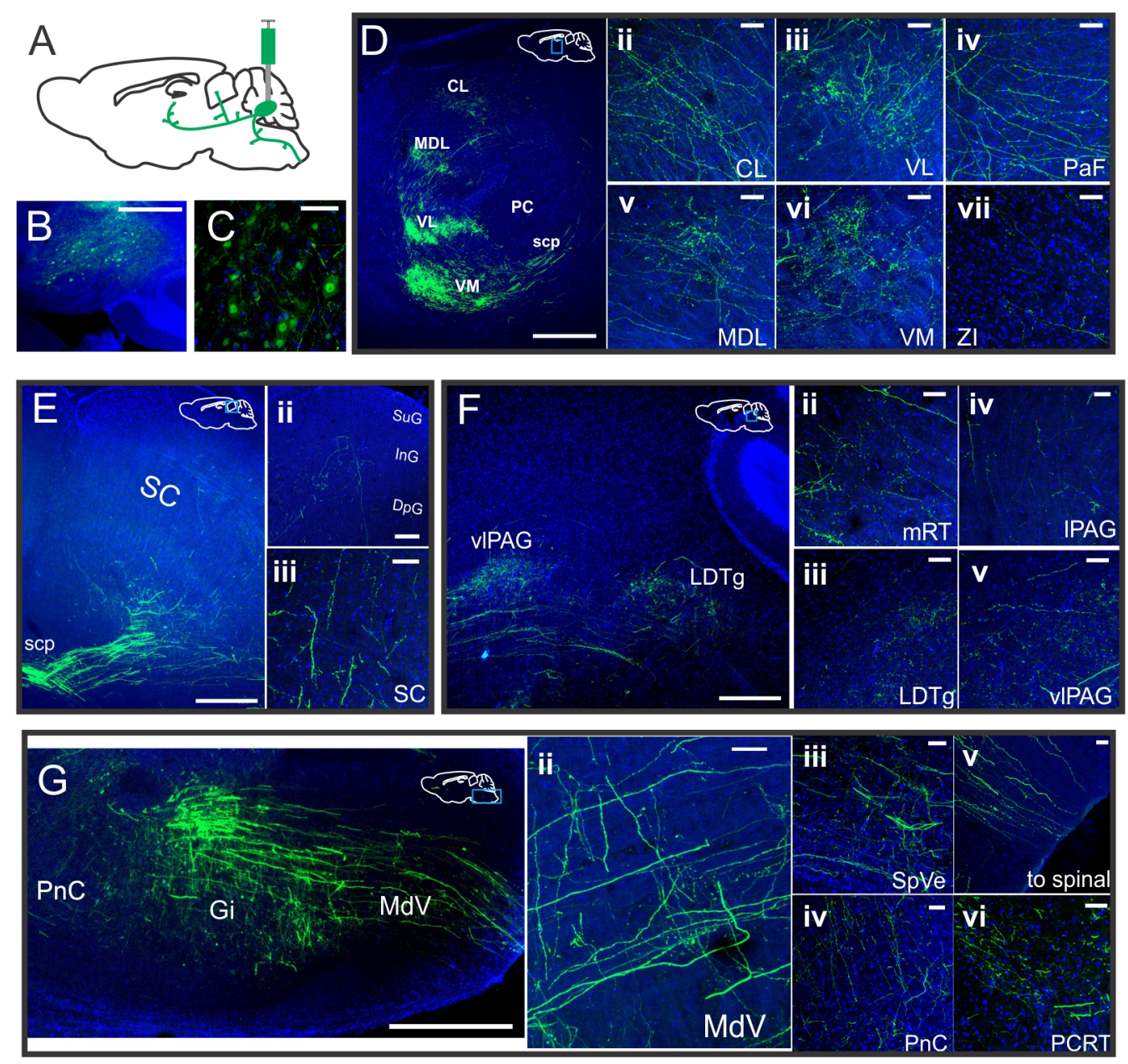

1020 Figure 1. Viral labeling illustrates a large number of downstream targets of fastigial neurons. A)

1021 Schematic of injection targeting, in which mice are injected with an AAV to induce the

1022 expression of GFP in fastigial neurons for broad labeling of fastigial outputs. B-C) GFP

1023 expression in fastigial neurons at two different magnifications. D) Six weeks post injection, GFP+

1024 fastigial fibers are visible in the thalamus (D) including central lateral (ii), ventral lateral (iii),

1025 parafascicular (iv), medial dorsal (v), ventral medial (vi) and zona incerta (vii) nuclei. Blue box

1026 over white schematic indicates approximate region of focus. E) Fastigial fibers are also visible in

1027 the superior colliculus, including both deep and intermediate layers (ii-iii). F) In the midbrain,

1028 fastigial fibers are observed in the mesencephalic reticular formation (ii), laterodorsal tegmental

1029 nucleus (iii), as well as both the lateral (iv) and ventral lateral (v) periaqueductal gray. G) 
1030 Extensive fastigial fibers are visible in the brainstem, including the medullary reticular formation

1031 (ii), vestibular nuclei including the spinal vestibular nucleus (iii), and pontine reticular nuclei (iv, 1032 vi). Fibers are also visible in more caudal regions of the brainstem, likely travelling to the spinal 1033 cord (v). CL: central lateral nucleus, MDL: medial dorsal nucleus, lateral part, VL: ventral lateral 1034 nucleus, VM: ventral medial nucleus, PC: paracentral nucleus, SC: superior colliculus, scp:

1035 superior cerebellar peduncle, PaF: parafascicular nucleus, ZI: zona incerta, SC: superior

1036 colliculus, $\mathrm{SuG}$ : superior gray layer, $\mathrm{InG}$ : intermediate gray layer, DpG: deep gray layer, vlPAG:

1037 ventral lateral periaqueductal gray, LDTg: laterodorsal tegmental nucleus, mRT: mesencephalic

1038 reticular nucleus, IPAG: lateral periaqueductal gray, PnC: nucleus reticularis pontis caudalis, Gi:

1039 gigantocellular reticular nucleus, MdV: medullary reticular nucleus (ventral part), SpVe: spinal

1040 vestibular nucleus, PCRT: parvocellular reticular nucleus. All images are taken from sagittal

1041 sections; see methods for approximate medial lateral coordinates for each region. Scale bars: 200

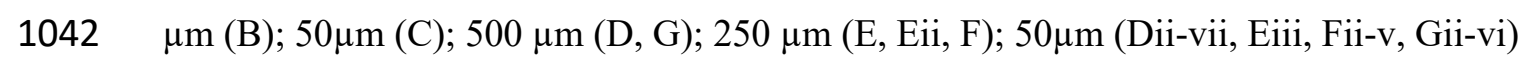



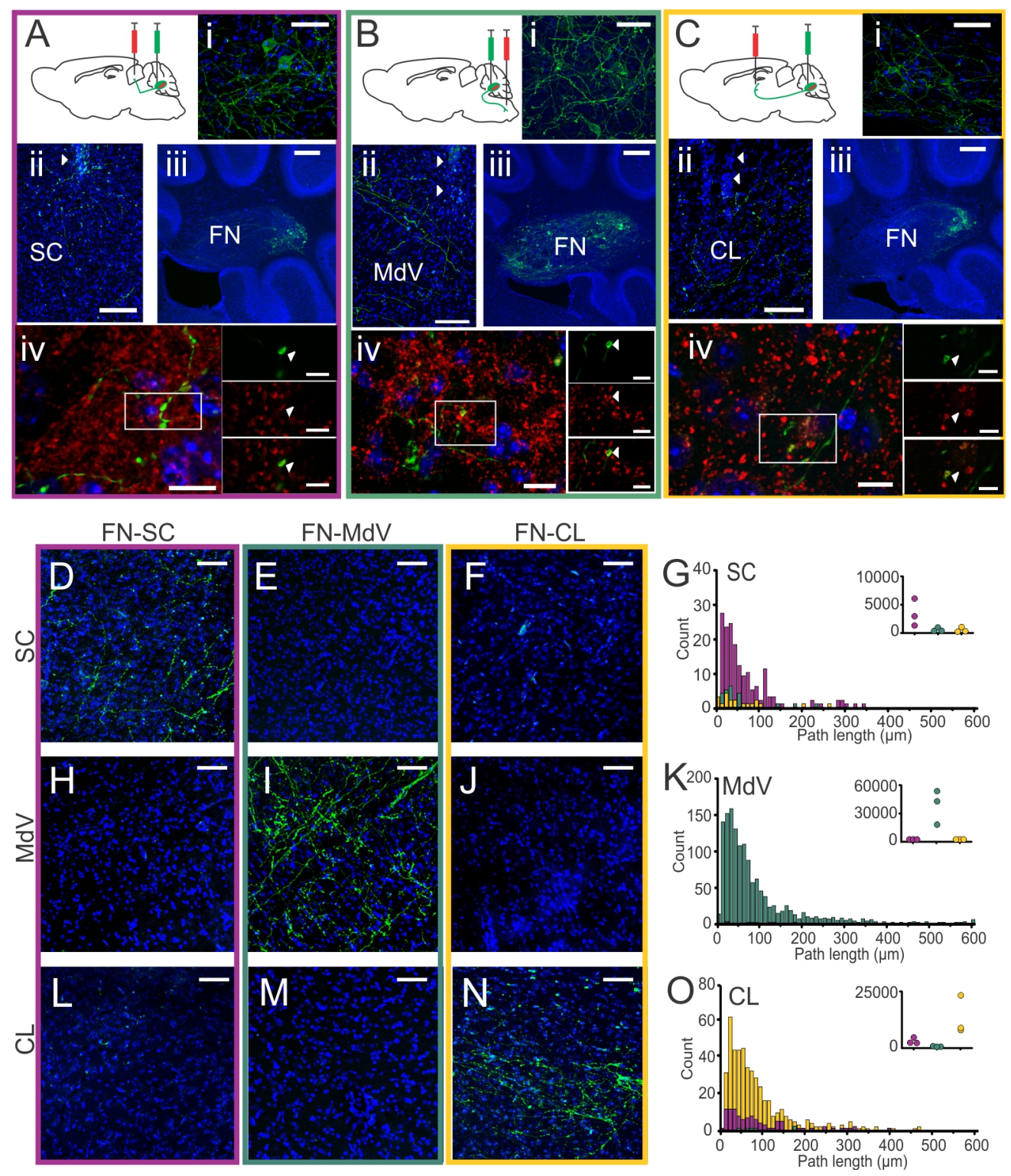

1046 Figure 2. A dual-virus targeting strategy reveals fastigial output channels to segregated

1047 projection targets. A) Schematic of injection targeting for FN-SC neurons. Mice are injected with

1048 a virus for retrograde expression of Cre (red syringe) in the superior colliculus followed by

1049 injection of a virus for Cre-dependent expression of ChR-eYFP in the fastigial nucleus (green

1050 syringe). Dual injection results in labeling of fastigial neurons (i) after injection to superior

1051 colliculus (ii, arrows denote injection tract). FN-SC cell bodies expressing ChR-eYFP are

1052 observed in the caudal fastigial nucleus (iii), and VGluT2+ (shown in red) fastigial fibers (green) 
1053 are present in the superior colliculus (iv). B) same as for (A), but for MdV injection targeting, in 1054 which fastigial cell bodies (i) are labeled after retro-virus injection in the MdV (ii). FN-MdV cell 1055 bodies expressing ChR-eYFP are observed in both rostral and caudal portions of the fastigial 1056 nucleus (iii), and VGluT2 + fastigial fibers are present in the MdV (iv). C) Same as for (A-B), but 1057 for CL retro-virus injection targeting. D-F) Extensive fastigial fibers are observed in the SC for 1058 FN-SC, but not FN-MdV nor FN-CL neurons. G) Histogram of path lengths in the SC for FN-SC 1059 (magenta), FN-MdV (teal), and FN-CL (yellow) neurons ( $\mathrm{n}=3$ animals per group, inset indicates 1060 total path lengths for each individual animal). H-J) Extensive fastigial fibers are observed in the 1061 MdV for FN-MdV, but not for FN-SC or FN-CL, neurons. K) Histogram of path lengths and inset 1062 dot plots of total path length for each individual animal in the MdV for FN-SC, FN-MdV, and

1063 FN-CL neurons. L-N) Dense fibers are observed in the CL for FN-CL, but not SC or MdV, 1064 neurons. O) Histogram of path lengths and inset dot plots of total path length for each individual 1065 animal in the CL for FN-SC, FN-MdV and FN-CL neurons. All images are taken from sagittal

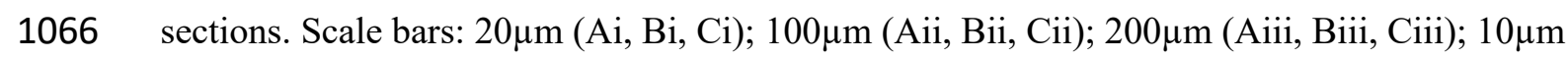
1067 (Aiv, Biv, Civ, insets $2 \mu \mathrm{m}) 50 \mu \mathrm{m}(\mathrm{D}-\mathrm{F}, \mathrm{H}-\mathrm{J}, \mathrm{L}-\mathrm{N})$. 

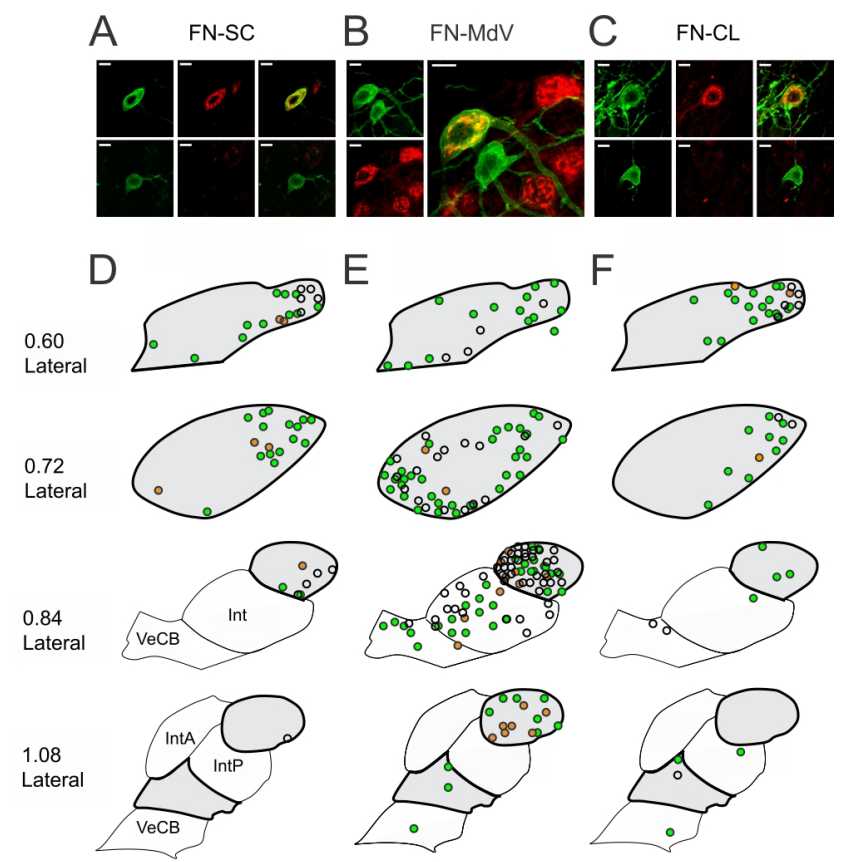

1070

Figure 3. Anatomical characterization of FN-SC, FN-MdV, and FN-CL neurons. A-C)

1071

Osteopontin (SPP1) is not a reliable marker of FN-SC, FN-MdV, nor FN-CL neurons. A)

1072 Example SPP1-immunopositive (top row; SPP1-immunolabeling in red, eYFP-ChR2 expression

1073 in green; right: colocalization) and SPP1-immunonegative (bottom row) FN-SC neuron. B)

1074 Example SPP1+ and SPP1- FN-MdV neurons. Note that SPP1+/eYFP+ (yellow in overlay),

1075 SPP1+/eYFP- (red in overlay), and SPP1-/eYFP+ (green in overlay) neurons are visible in the

1076 same location (field of view) within the FN. C) Example SPP1+ (top row) and SPP1- (bottom

1077 row) FN-CL neurons. D-F) FN-SC neurons (D) and FN-CL neurons (F) are primarily located in

1078 the caudal FN, whereas FN-MdV neurons (E) are found in both rostral and caudal portions. FN

1079 denoted by thick outline and gray shading. Circles denote eYFP labeled cells $(n=3$ animals

1080 total). For tissue assessed for SPP1 ( $n=2$ animals each), eYFP cells displaying SPP1+

1081 immunolabeling are filled orange, and eYFP cells negative for SPP1-immunolabeling are filled

1082 green. Scale bars: $10 \mu \mathrm{m}$. Int: interposed nucleus, IntA: Interposed nucleus, anterior part, IntP:

1083 interposed nucleus, posterior part, $\mathrm{VeCB}$ : vestibulocerebellar nucleus. 


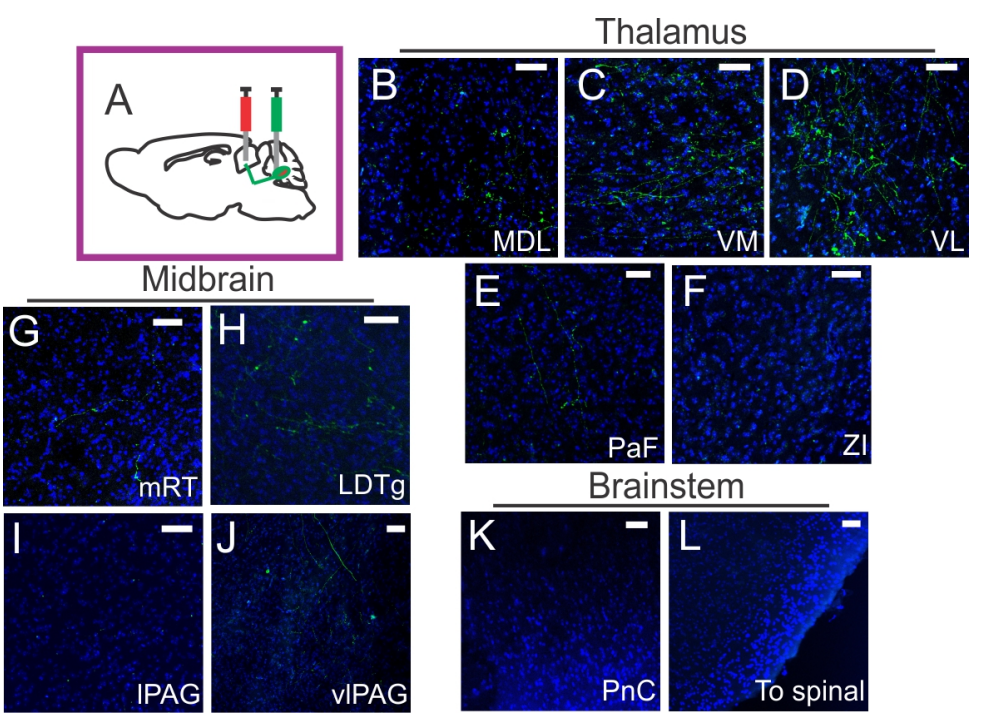

1086 Figure 4. FN-SC neurons send collaterals to distinct thalamic and midbrain nuclei. A) Schematic

1087 of injection targeting for FN-SC neurons. In addition to strong fibers in the SC (Figure 2D), FN-

1088 SC neurons display some fibers in the thalamus (B-F), especially in the VL nucleus (D). F) No

1089 fibers are observed in the zona incerta (ZI). In the midbrain, sparse fibers are observed in some

1090 regions including the mRT $(\mathrm{G}), \operatorname{LDTg}(\mathrm{H})$, and vlPAG $(\mathrm{J})$. Little to no fibers are observed in

1091 IPAG (I) or the brainstem, including the PnC (K). There is also no evidence of FN-SC neurons

1092 sending fibers to the spinal cord (L). All images are taken from sagittal sections. Abbreviations

1093 are as for Fig. 1. Scale bars $50 \mu \mathrm{m}$. 

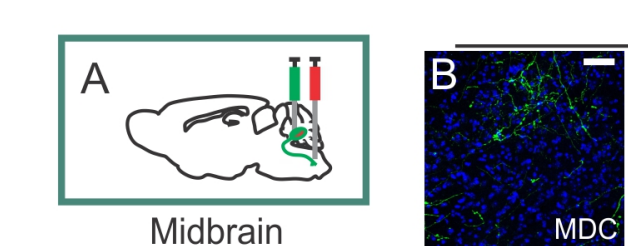

Thalamus
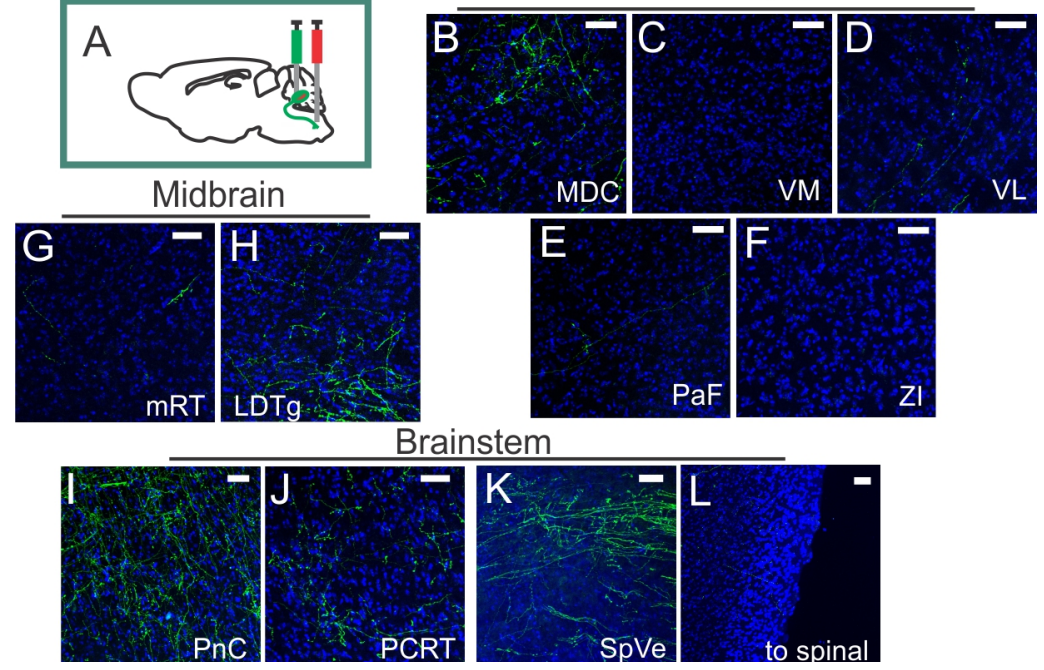

Figure 5. Collaterals from FN-MDV neurons have strong projections to several areas, but are

1098 largely restricted to the midbrain and brainstem. A) Schematic of injection targeting for FN-MdV

1099 neurons. B-F) FN-MdV fibers in the thalamus are almost entirely limited to the central portion of

1100 the medial dorsal nucleus (B), with little to no FN-MdV fibers observed in other thalamic nuclei

1101 (C-F). G-H) In the midbrain, sparse fibers are observed in the mRT (G), with stronger expression

1102 observed in the LDTg (H). I-L) In the brainstem, extensive fibers are observed in regions

1103 including the PnC (I) and PCRT (J), as well as vestibular nuclei, including the SpVe (K). As was

1104 noted for FN-SC neurons, there is little evidence of FN-MdV neurons projecting to the spinal

1105 cord (L). MDC: Medial dorsal nucleus, central portion. All other abbreviations are as for Fig. 1.

1106 All images are taken from sagittal sections. Scale bars $50 \mu \mathrm{m}$. 

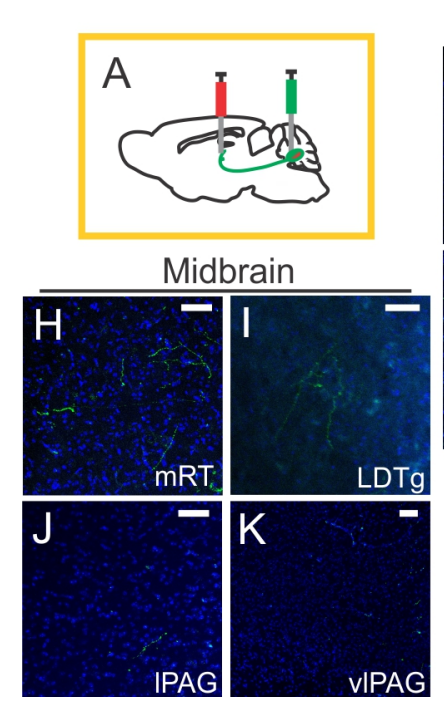

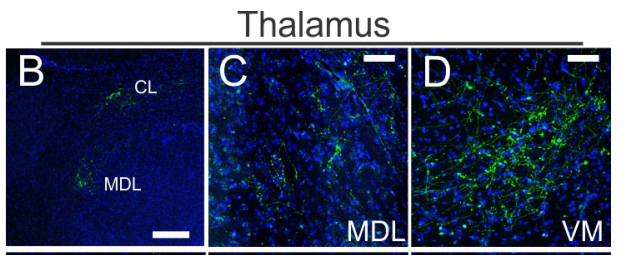

Figure 6. Collaterals from FN-CL neurons are observed in additional thalamic nuclei. A)

1113 Schematic of injection targeting for FN-CL neurons. B-G) In addition to the CL nucleus, FN-CL

1114 neurons also send projections to other thalamic nuclei, including MDL (C), and VM (D) thalamic

1115 nuclei. Sparse fibers are also observed in the $\mathrm{PaF}(\mathrm{E})$ and $\mathrm{ZI}(\mathrm{G})$. Note that panel B provides a

1116 lower magnification image of the thalamus, illustrating the patches of expression in the CL and

1117 MDL. H-K) Limited expression is observed in the midbrain. L) Essentially no fibers are

1118 observed in the brainstem, including PnC. L) As with FN-SC and FN-MdV neurons, there is no

1119 evidence of FN-CL neurons sending projections to the spinal cord. Abbreviations are as for Fig.

1120 1. All images are taken from sagittal sections. Scale bars $50 \mu \mathrm{m}$. 

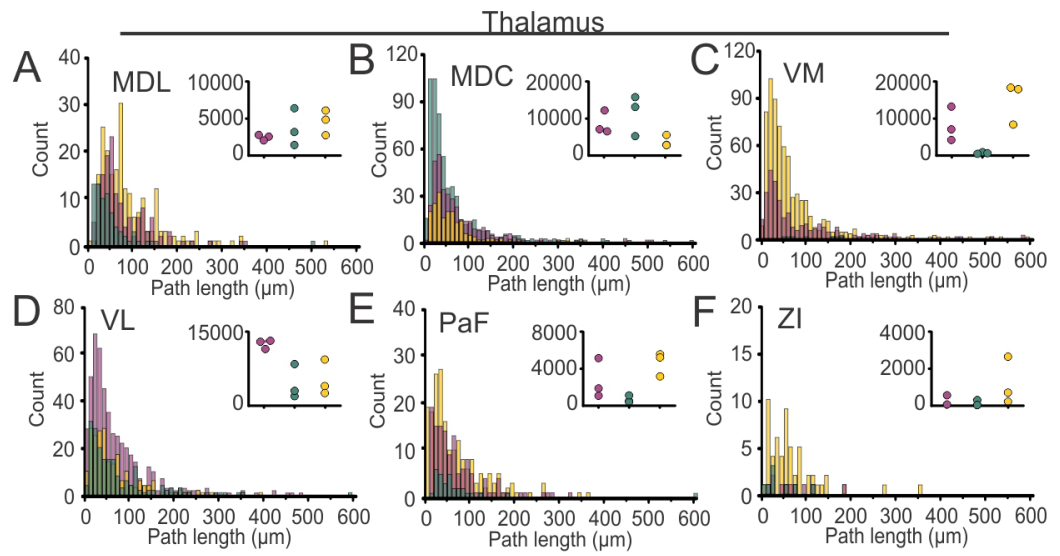

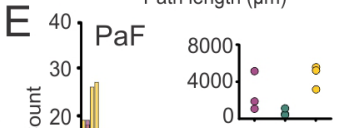

$\mathrm{F}^{20} \mathrm{ZI}$
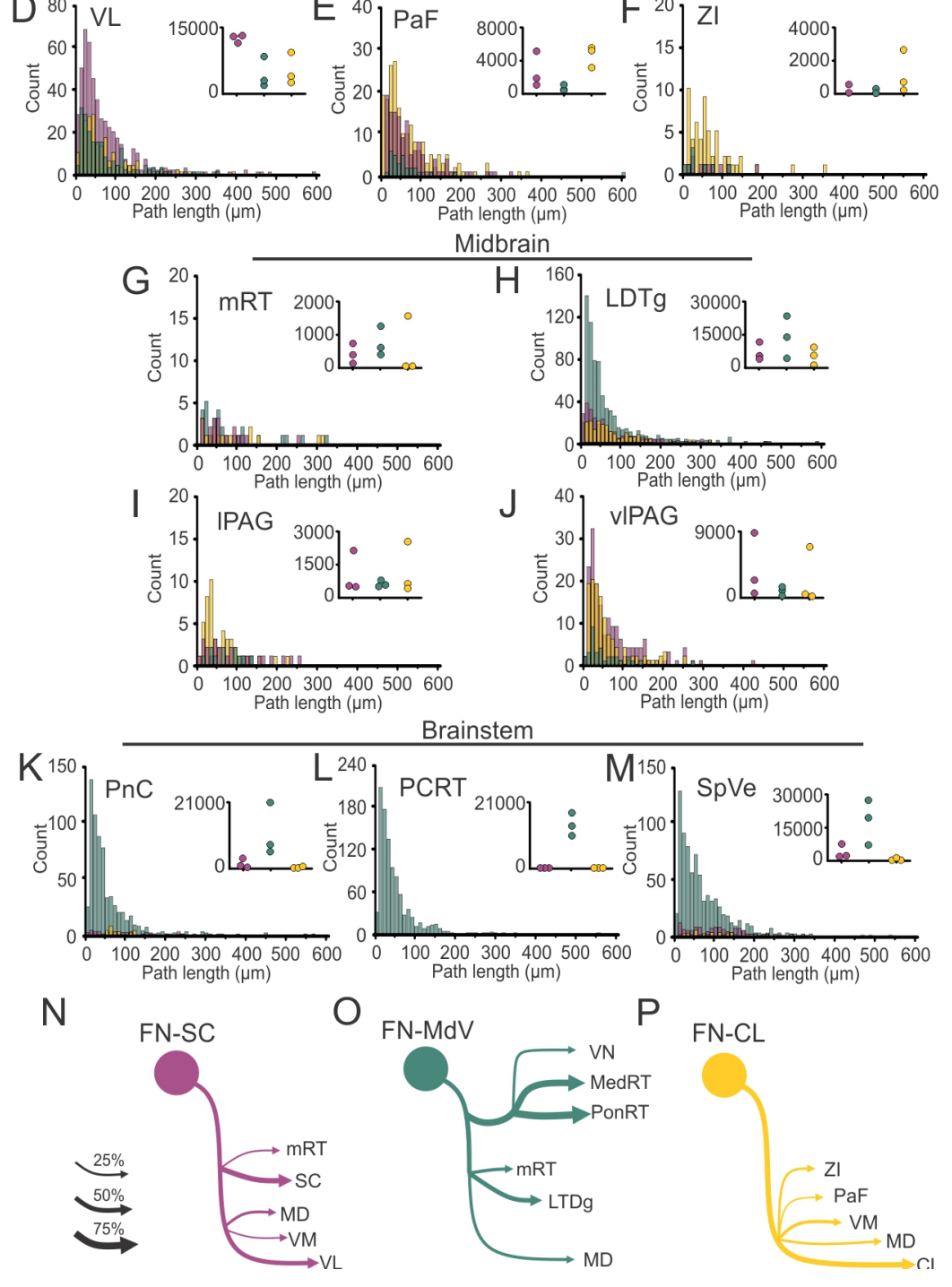

160

Figure 7. Fastigial outputs form largely segregated, but somewhat overlapping, channels. A-F)

1128 Histograms of path lengths of fibers in thalamic (A-F), midbrain (G-J), and brainstem nuclei (K-

1129 M) for FN-SC (magenta), FN-MdV (teal), and FN-CL (yellow) neurons ( $\mathrm{n}=3$ animals per group,

1130 dot plot insets indicate total path length for each animal across all groups). Note differences in y-

1131 axis scales for different regions. N-P) Schematic illustrations of brain regions receiving relatively

1132 strong inputs from FN-SC (N), FN-MdV (O), and FN-CL (P) neurons, with line thickness 
1133 roughly indicating relative strength, compared to max-observed fastigial inputs to that area. VN:

1134 Vestibular nuclei including SpVe, SuVE, and MVe; note that quantification was only done for

1135 SpVe. MedRT: Medullary reticular nuclei including Gi and MdD; note that quantification was

1136 only done for MdV. PonRT: Pontine reticular nuclei including PnC, PnO, PCRT, and PCRTa;

1137 note that quantification was only done for PnC and PCRT. All other abbreviations are as for Fig.

$1138 \quad 1-6$.

1139

1140

1141 
A FN-SC
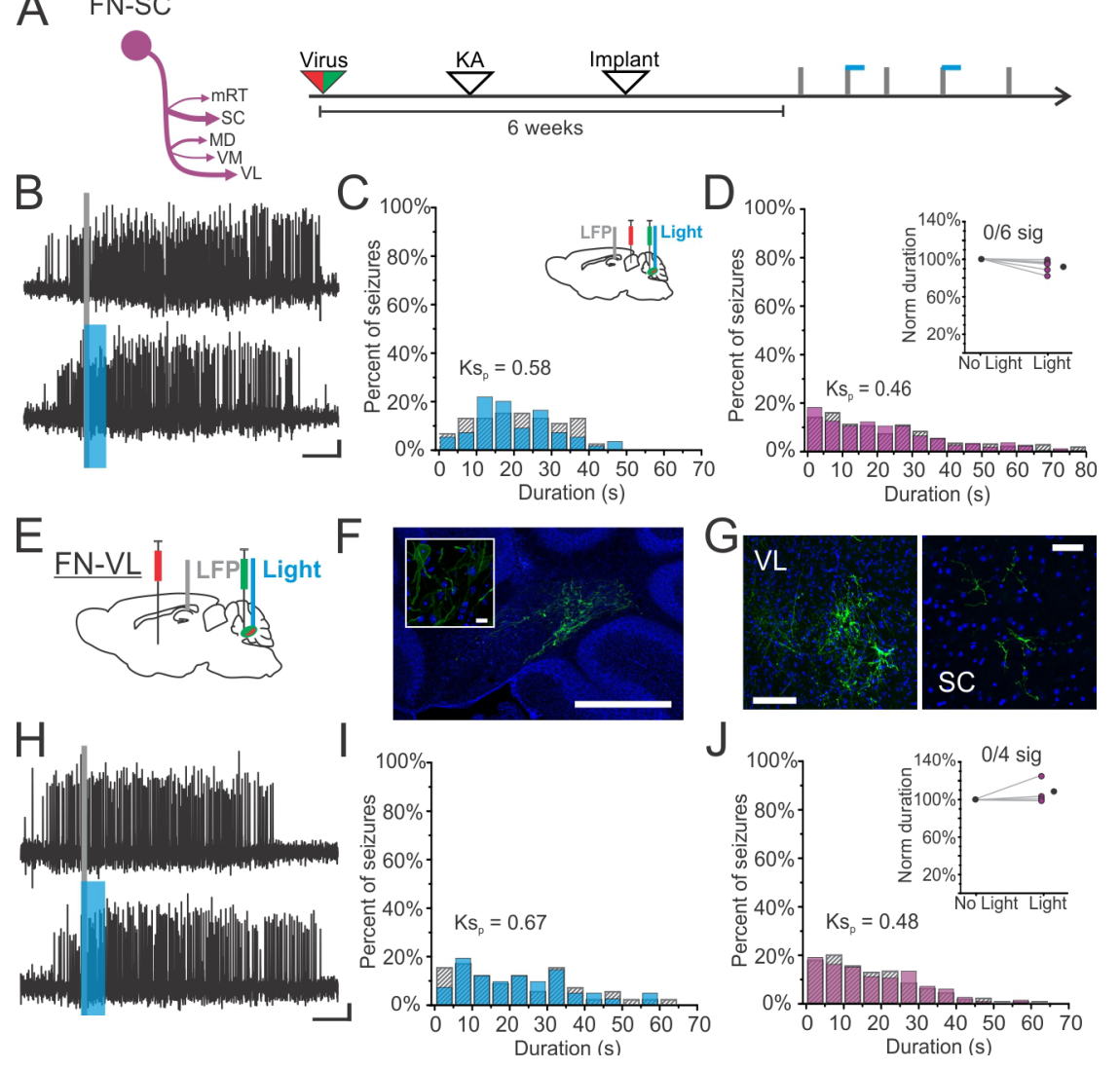

Figure 8. On-demand optogenetic excitation of FN-SC neurons fails to attenuate hippocampal seizures. A) FN-SC output channel and experimental design schematics. Mice received dual viral

1147 injections and underwent epilepsy induction, followed by implantation of electrodes in the

1148 hippocampus and optic fibers in the fastigial nucleus for targeting FN-SC. B) Example seizure

1149 events detected (gray line) on-line that were randomly elected to either not receive light (top

1150 trace) or receive on-demand light delivery (bottom trace) to FN-SC neurons. Blue bar indicates

1151 timing of light delivery. C) Histograms of post-detection seizure durations for events not

1152 receiving light (hashed bars) versus those receiving light (blue bars) in an example animal with

1153 targeting of FN-SC neurons, illustrating no significant effect of light delivery on hippocampal

1154 seizure duration ( $\mathrm{p}=0.58$, two sample Kolmogorov-Smirnov test). Inset indicates experimental

1155 schematic of viral targeting, electrode recording, and optogenetic stimulation. D) Population

1156 histograms of post-detection seizure durations across FN-SC mice ( $n=600$ events, from six 
1157 animals), showing no significant effect of light delivery ( $\mathrm{p}=0.46$, two sample Kolmogorov-

1158 Smirnov test). Inset: 0 out of 6 FN-SC mice ( $p>0.05$, two sample Kolmogorov-Smirnov test)

1159 show a significant effect of light delivery (black circle indicates mean). E) Additional targeting

1160 strategy, using retrograde viral injection into the VL nucleus of the thalamus. F) Dual viral

1161 targeting of FN-VL neurons labels neurons in the caudal portion of the fastigial nucleus (inset

1162 shows higher magnification image), as well as fastigial fibers in both the VL nucleus (G, left) and

$1163 \mathrm{SC}(\mathrm{G}$, right). H) Example seizure events detected (gray line) on-line that were randomly elected

1164 to either not receive light (top trace) or receive on-demand light delivery (bottom trace) to FN-VL

1165 neurons. I) Seizure durations from an example animal with targeting of FN-VL neurons,

1166 illustrating no significant effect of light delivery $(\mathrm{p}=0.67$, two sample Kolmogorov-Smirnov

1167 test). J) Population histograms of post-detection seizure durations across FN-VL mice ( $\mathrm{n}=400$

1168 events, from four animals), showing no significant effect of light delivery ( $p=0.48$, two sample

1169 Kolmogorov-Smirnov test). Inset: 0 out of 4 FN-VL mice show a significant effect of light

1170 delivery. Scale bars: $5 \mathrm{sec}, 0.05 \mathrm{mV}(\mathrm{B}, \mathrm{H}) ; 500 \mu \mathrm{m}(\mathrm{F}$, inset $10 \mu \mathrm{m}) ; 100 \mu \mathrm{m}(\mathrm{G}$, left); $50 \mu \mathrm{m}(\mathrm{G}$,

1171 right).

1172 

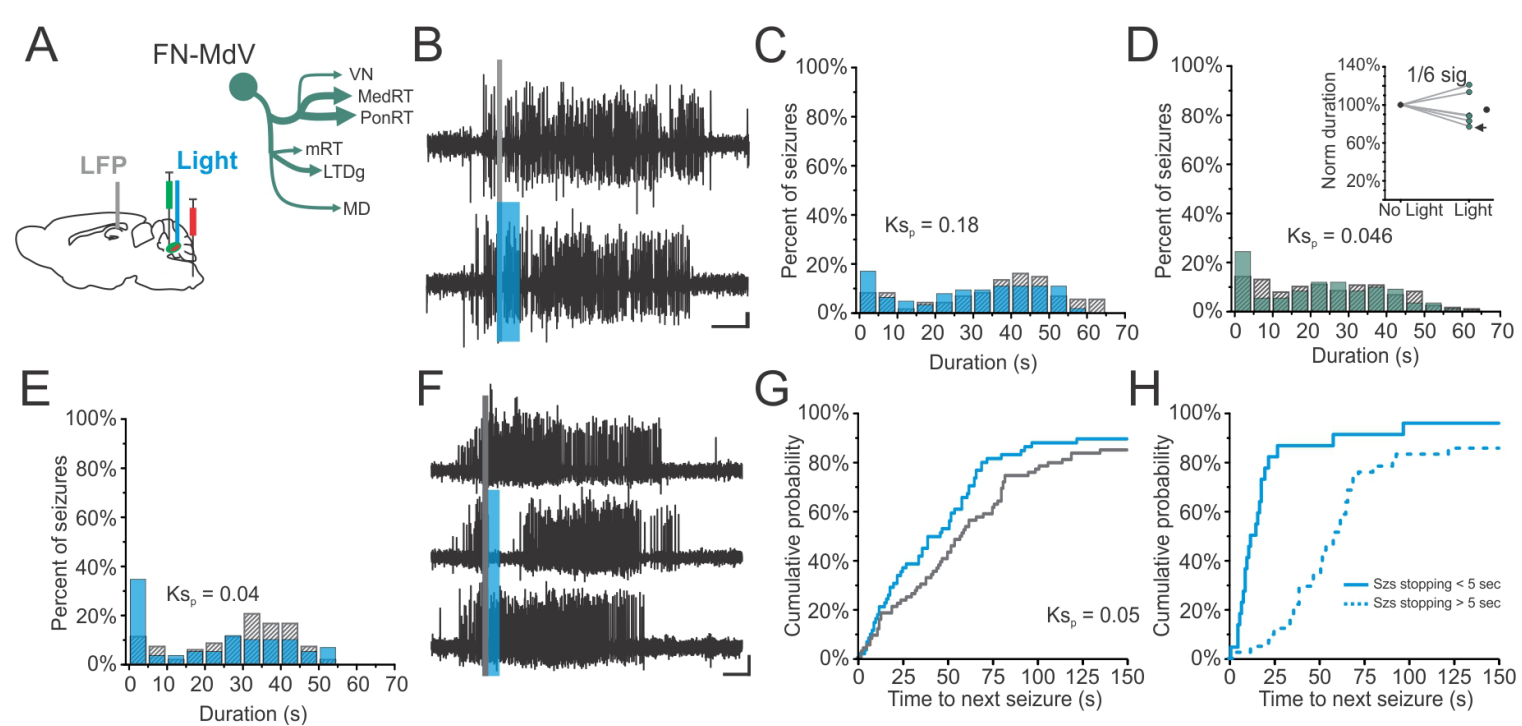

1174 Figure 9. On-demand optogenetic excitation of FN-MdV neurons fails to attenuate hippocampal

1175 seizures. A) FN-MdV output channel and experimental schematic for targeting. B) Example

1176 seizure events detected (gray line) on-line that were randomly elected to either not receive light

1177 (top trace) or receive on-demand light delivery (bottom trace) to FN-MdV neurons. Blue bar

1178 indicates timing of light delivery. C) Seizure durations from an example animal with targeting of

1179 FN-MdV neurons, illustrating no significant effect of light delivery $(p=0.18$, two sample

1180 Kolmogorov-Smirnov test). D) Population histograms of post-detection seizure durations across

1181 FN-MdV mice ( $\mathrm{n}=600$ events, from six animals), showing a marginally significant effect of light

1182 delivery $(\mathrm{p}=0.046$, two sample Kolmogorov-Smirnov test). Inset: 1 out of 6 FN-MdV mice show

1183 a significant effect of light delivery (indicated by arrow). E) Histograms of post-detection seizure

1184 durations for events not receiving light (hashed bars) versus those receiving light (blue bars) in

1185 the one FN-MdV animal showing a significant effect of light delivery $(p=0.04$, two sample

1186 Kolmogorov-Smirnov test; $23 \%$ reduction in post-detection seizure duration with light in this

1187 animal). F) Example seizure events that were randomly elected to either not receive light (top

1188 trace) or receive light delivery (middle and bottom traces) to FN-MdV neurons in that one animal,

1189 illustrating that the effect of light delivery, when present, is more of a pause in seizures than a

1190 true attenuation. G) Cumulative probabilities of time to next seizure for seizure events receiving 
1191 light (blue trace) and those not receiving light (gray trace), illustrating a trend towards a decrease

1192 in time to next seizure for events receiving light in this animal $(\mathrm{p}=0.05$, two sample

1193 Kolmogorov-Smirnov test; overall, a 13\% reduction in time to next seizure). H) Seizure events

1194 which received light and stopped within 5 seconds tended to have a shorter time to next seizure

1195 (solid blue line) versus those that did not stop within 5 seconds (dotted blue line), again

1196 suggesting brief interruptions to seizures, rather than robust inhibition in this animal. Note that no

1197 light seizure events are not plotted, as only 12 no light seizures lasted less than 5 seconds. Scale

1198 bars: $5 \mathrm{sec}, 0.05 \mathrm{mV}(\mathrm{B}, \mathrm{F})$.

1199 

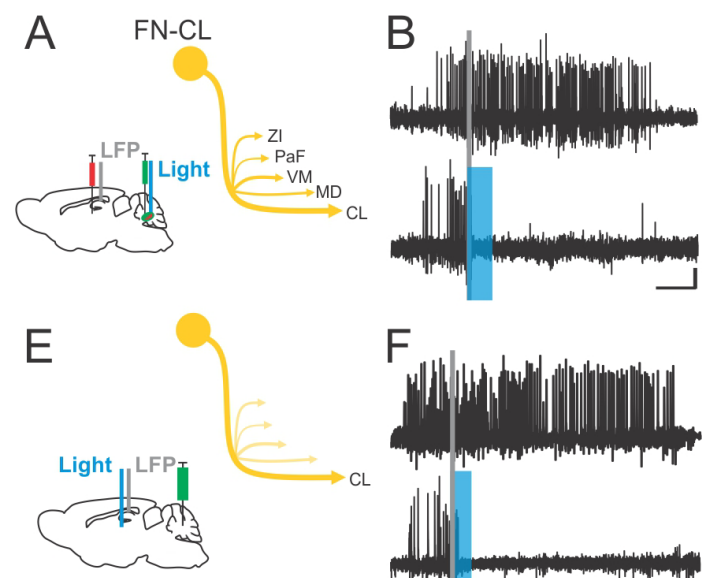

$\mathrm{F}$

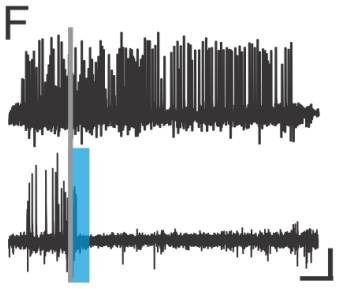

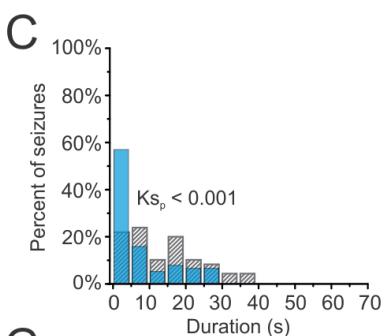

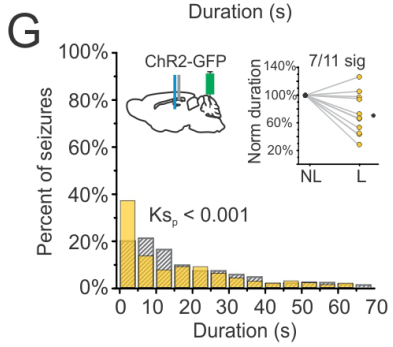

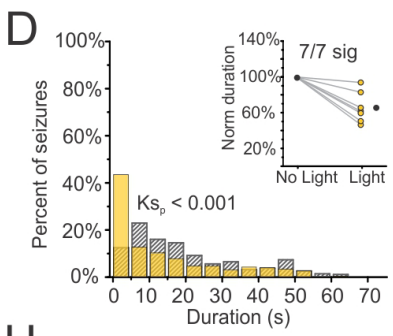

$\mathrm{H}$

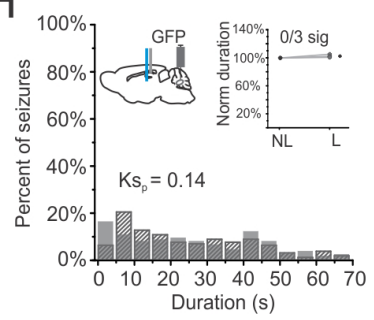

1201 Figure 10. On-demand optogenetic activation of FN-CL neurons robustly attenuates hippocampal seizures. A) FN-CL output channel and experimental schematic for targeting. B) Example seizure events detected (gray line) on-line that were randomly elected to either not receive light (top trace) or receive on-demand light delivery (bottom trace) to FN-CL neurons. Blue bar indicates timing of light delivery. C) Seizure durations from an example animal with targeting of FN-CL neurons, showing a significant effect of light delivery $(\mathrm{p}<0.001$, two sample Kolmogorov-

1207 Smirnov test). D) Population histograms of post-detection seizure duration events across FN-CL 1208 mice ( $\mathrm{n}=700$ seizure events, from seven mice), showing a significant effect of light delivery $(\mathrm{p}<$ 12090.001 , two sample Kolmogorov-Smirnov test). Inset: Normalized seizure duration for light versus 1210 no light across FN-CL mice, with 7 out of 7 mice (100\%) showing a significant reduction at the 1211 animal level. Black dot denotes average. E) To directly target FN fibers in the CL nucleus, mice

1212 were injected in the fastigial nucleus with virus encoding channelrhodopsin, followed by

1213 intrahippocampal kainic acid for induction of epilepsy, and then implanted with electrodes in the 1214 hippocampus and optic fibers targeting the central lateral nucleus for on-demand interventions. F)

1215 Example detected seizure events that were randomly elected to either not receive light (top trace)

1216 or receive light delivery (bottom trace) to fastigial fibers in the CL nucleus. Blue bar indicates

1217 timing of light delivery. G) Population histograms of post-detection seizure duration events

1218 across FN-CL mice ( $\mathrm{n}=1100$ seizure events, from eleven mice), showing a significant effect of 
1219 light delivery $(\mathrm{p}<0.001$, two sample Kolmogorov-Smirnov test). Inset: Normalized seizure

1220 duration for light versus no light across CL fiber targeting mice, with 7 out of 11 mice showing a

1221 significant reduction at the animal level. Block dot indicates average. H) No effect of light

1222 delivery to the CL is observed in control animals $(n=300$ seizure events from 3 mice, $p=0.14$,

1223 two sample Kolmogorov-Smirnov test). Scale bars: $5 \mathrm{sec}, 0.05 \mathrm{mV}$ (B, F).

1224 